\title{
Le immagini negate
}

\author{
Adriana Rossi \\ Umberto Palmieri
}

Abstract

Nell'ambito delle finalità proposte dal laboratorio di modelli reali funzionanti (mww.archeotecnica. $\mathrm{com})[\mathrm{I}]$ si è inteso promuovere e valorizzare le ricerche di ingegneri, architetti e designer, esperti di rilievo e rappresentazione (ICAR/ /7), di progettazione esecutiva di macchine (ING-IND// 4 e I5), di tecnologia (ICAR I2) e allestimenti museali (ICAR/I4) nella consapevolezza diacronica di eventi e evoluzioni storiche (ICAR/I8): campi in cui la comunicazione si è sempre servita del linguaggio del disegno tecnico. L'evoluzione di hardware e software ha permesso, poi, di estendere il significato tradizionale del 'disegno' a quello di 'modello' le cui caratteristiche, grazie ai CAD avanzati, travalicano la forma geometrica per comprendere tutti gli aspetti informativi atti a anticipare le costruzioni di cantiere con un livello di controllo e verifica prima impensabili. Qui si presentano alcune ricerche applicate.

Parole chiave

rilievo, progettazione museale, catapulta, patrimonio culturale, turismo sostenibile.

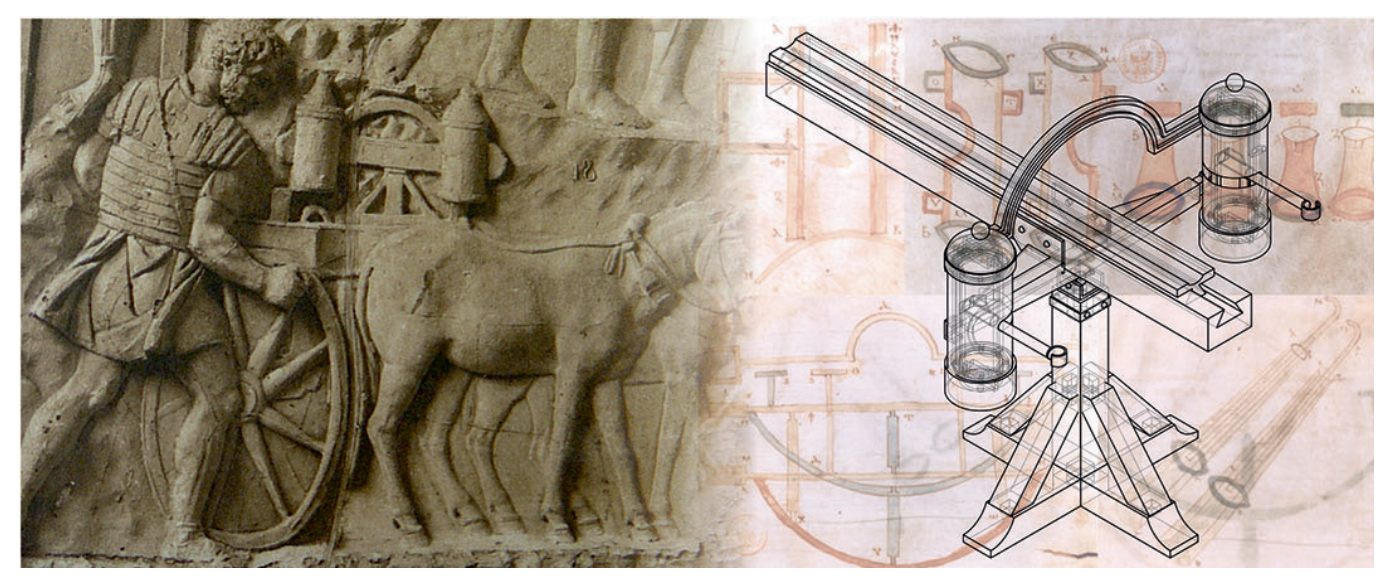




\section{Introduzione}

Probabilmente si trattò di un caso o, più verosimilmente, di una precisa circostanza, dal momento che anche ai Romani non sfuggivano le ricorrenze fatali. Esattamente cento anni dopo la disfatta di Teutoburg (9 d.C.) [2] si progettò il monumento celebrativo più vistoso della pur sterminata produzione imperiale: la Colonna Traiana. Inaugurata nel I I3, esaltava l'esercito e il suo capo supremo; lungo le sue spire si avvicendano I 50 'inquadrature' inerenti agli episodi salienti della guerra contro la Dacia (I01-106) [Rossi I98I], sfondo adeguato per l'enfatizzazione delle più avanzate capacità tecnico-militari di una civiltà superiore, come poco prima Plinio il Giovane aveva menzionato in una sua lettera: "Dices immissa terris nova flumina, novos pontes fluminibus iniectos, insessa castris montium abrupta, pulsum regia pulsum etiam vita regem nihil desperantem; super haec actos bis triumphos, quorum alter ex invicta gente primus, alter novissimus fuit"' [C. Plinii Caecilii Secundi Epistolae, VII, 2] [3].

Fra quei tanti 'fotogrammi' ne spiccano una decina che hanno per soggetto un tipo di armamento balistico fino ad allora ignoto, o non divulgato così platealmente, destinato ad avere, da quel momento in poi, un ruolo basilare nell'esercito romano. Si tratta di pezzi di artiglieria leggera o lancia-dardi, propriamente detti 'catapulte' e volgarmente 'scorpioni', riprodotti in tutti i diversi allestimenti: da fortezza, campale, ippotrainato leggero e ippotrainato pesante [Russo 2002, pp. 232-243].

Sebbene l'attuale età possa, a giusta ragione, definirsi 'delle immagini', non per questo le precedenti ne furono avare. In particolare, quella classica, ce ne ha tramandate una quantità innumerevole ed estremamente variegata, in grado di fornire un apporto basilare alla conoscenza del passato, forse il più oggettivo ed attendibile. Ovvio perciò equiparare quelle fonti iconiche alle tradizionali: ogni aspetto della vita, ogni evento rimarchevole reale o mitologico, ogni personaggio significativo meritò, infatti, una qualche raffigurazione, spesso su di un precipuo supporto. Tanto per esemplificare: le monete, di ampia diffusione e considerevole

Fig. I. Frammento della Colonna Traiana, Foro di Roma, Il sec. (110-113). I bassorilievi rievocando tutti i momenti salienti della campagna contro i Daci. In primo piano lo Scorpione attribuito all'ingegno di Apollodoro di Damasco (50/60130). Individuazione del modulo-modiolo. Dimensionamento riferito alle misure dettate da Eurone di Alessandria in Cheiroballista, $62 \mathrm{~d}$.C.

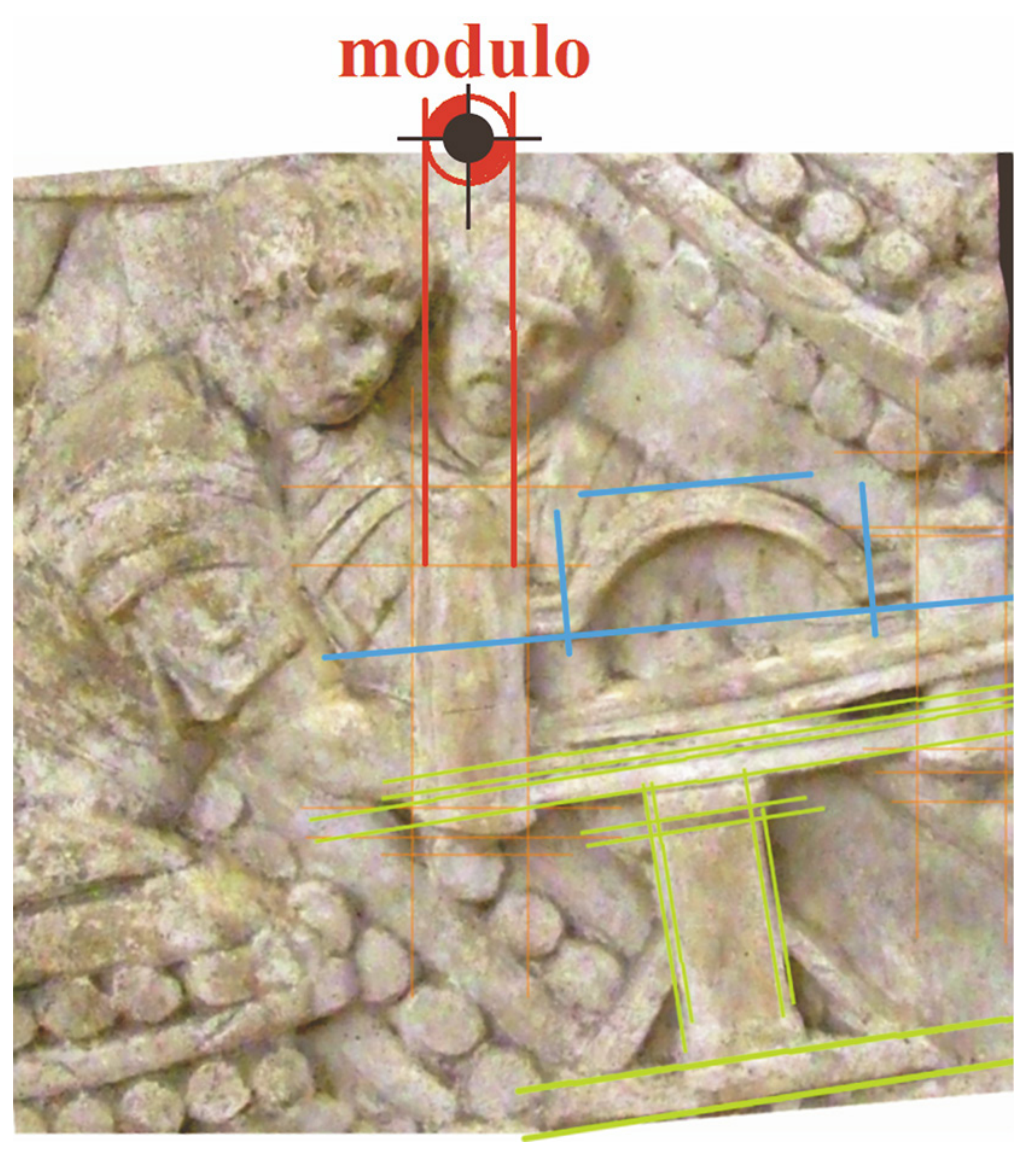


solidità, si ritennero ideali per ricevere i ritratti dei potenti o gli emblemi del potere; le pareti delle abitazioni, di vasta superficie e ben riparate dalle intemperie, lo furono per affreschi di paesaggi o di soggetti mitologici; i bassorilievi marmorei, insensibili agli oltraggi del tempo, vennero prescelti per le rievocazioni celebrative; statue di pietra e di bronzo per la raffigurazione di personaggi, umani o divini, da proporre all'ammirazione o al culto dei posteri; gioielli e gemme per incisioni e figurine scaramantiche, allegoriche od erotiche. Sensato concludere che i nostri progenitori non hanno trascurato nulla della loro esistenza tramandandocene per conseguenza ogni aspetto.

Accanto alla realtà, per quanto detto, abbondano immagini fantastiche, mitologiche, leggendarie: un'orgia di divinità, di centauri, di chimere, di satiri e di mostri di vario tipo popolano quelle narrazioni iconiche. Non difettano inoltre scene epiche con combattimenti terrestri e navali, assedi e stragi. Qualsiasi cosa le fonti scrivano, diventa possibile per noi - con un po' di pazienza - trovare l'immagine corrispettiva. Precisazione che sembra stringente soprattutto nell'ambito della tecnologia, dell'artigianato, delle costruzioni in genere essendo per loro intrinseca natura le vere novità del pianeta e della cultura.

Fig. 2. 'Archotenica' di Flavio Russo.Visita alle officine a.a.I6-I7 LabTAR Tecniche Avanzate della Rappresentazione | Prodotto prof.Adriana Rossi LMI2 Dip. Ingegneria, Luigi Vanvitelli).
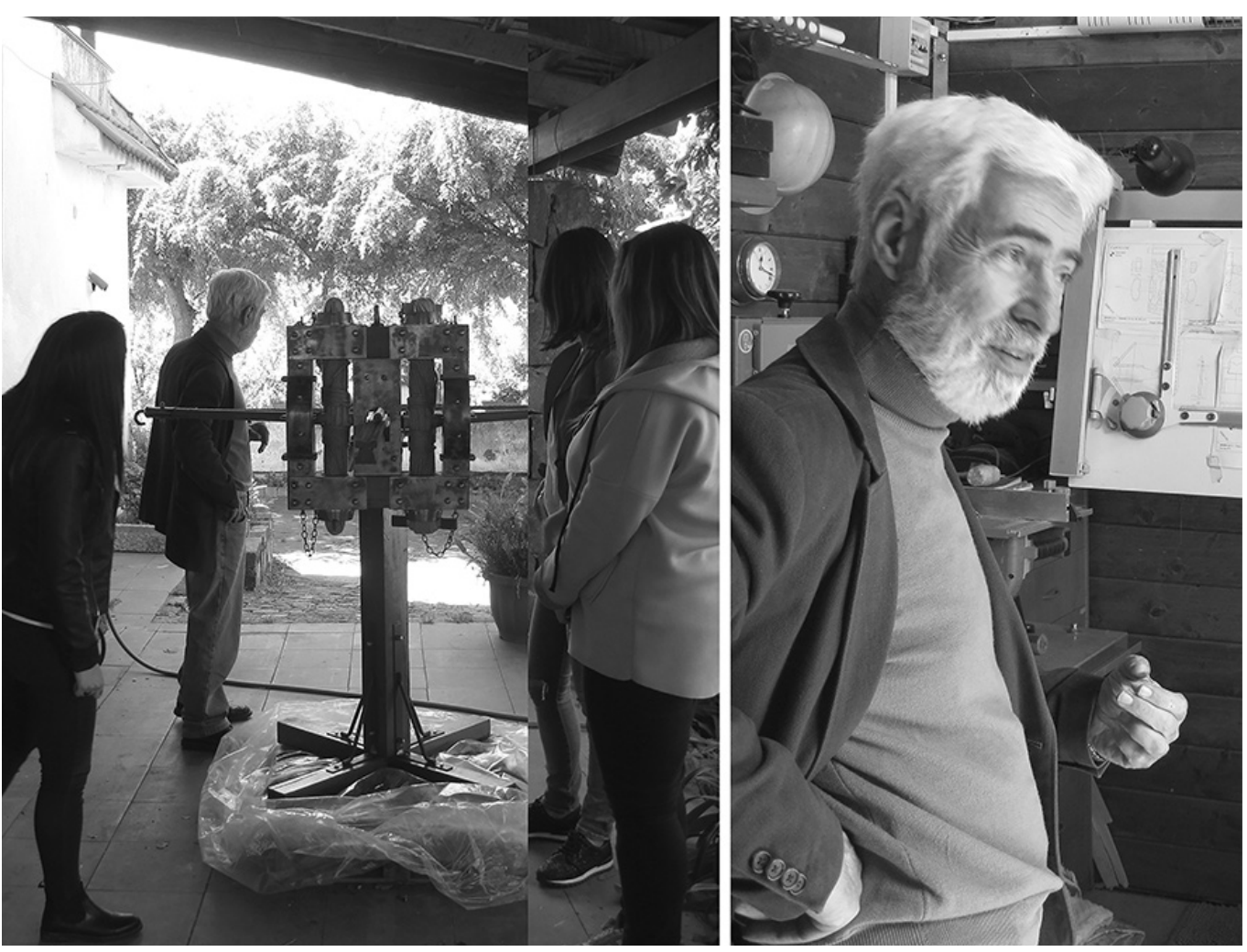

\section{Gli armamenti balistici}

È certamente significativo che i Romani definirono ingegna le macchine impiegate negli assedi per infrangere la difesa nemica: in pratica l'ampia gamma di congegni per scavalcare le mura o per abbatterne i difensori da notevole distanza. Più tardi, con l'avvento delle macchine a torsione, ribattezzarono quelle da lancio tormenta. Macchine sempre di notevole complessità alle quali, però, forse per il disprezzo che circondava il lavoro manuale proprio degli schiavi, i coevi dedicarono scarsissime descrizioni e meno ancora immagini [Russo 2004, pp. 199-280].

Aristotele, dando una ennesima prova del suo raffinato raziocino, contestando le elucubrazioni filosofiche di Platone, affermò che la guerra avrebbe dovuto avvalersi non solo 
della virtù fisica ma anche di quella tecnica, della forza e dell'ingegno, facendo un esplicito riferimento alle artiglierie, delle quali ricorda le ormai conseguite precisione e potenza. Paradossalmente tanto nel breve periodo precedente quanto nel lunghissimo successivo, oltre un millennio, pochissimi però sentirono la necessità di fornire di quelle armi una dettagliata descrizione e, meno che mai, una raffigurazione.

Macchine di cui, infatti, non abbiamo nessuna immagine esplicita, al punto di dubitare persino della loro concreta esistenza. Per la verità ne abbiamo di parziali, limitate cioè a un dettaglio del loro propulsore: per l'esattezza, ne abbiamo appena due in oltre due secoli d'impiego! [4]. Come dire due incerte rappresentazioni parziali del motore di un autoveicolo, quando ormai ne circolano a milioni da più di un secolo! Perché gli antichi congiurarono a quel silenzio quasi totale ed assoluto? Perché non vollero tassativamente tramandarci quelle loro conquiste meccaniche? Perché non se ne sentirono mai orgogliosi da raffigurarle in qualche modo? E quando finalmente ne ritrassero alcune sulla Colonna Traiana, il loro propulsore a due cilindri restò talmente enigmatico da suggerire le più assurde elucubrazioni: come collocare poderose molle elicoidali in un quadrello d'acciaio da $16 \mathrm{~mm}$, all'interno degli stessi cilindri? Peccato che i romani non conoscessero né le molle elicoidali né l'acciaio massiccio! La prima spiegazione che viene in mente, insiste su una prassi squisitamente militare: tenere gelosamente segreta ogni arma di rivoluzionaria consequenzialità bellica. Segreto militare,
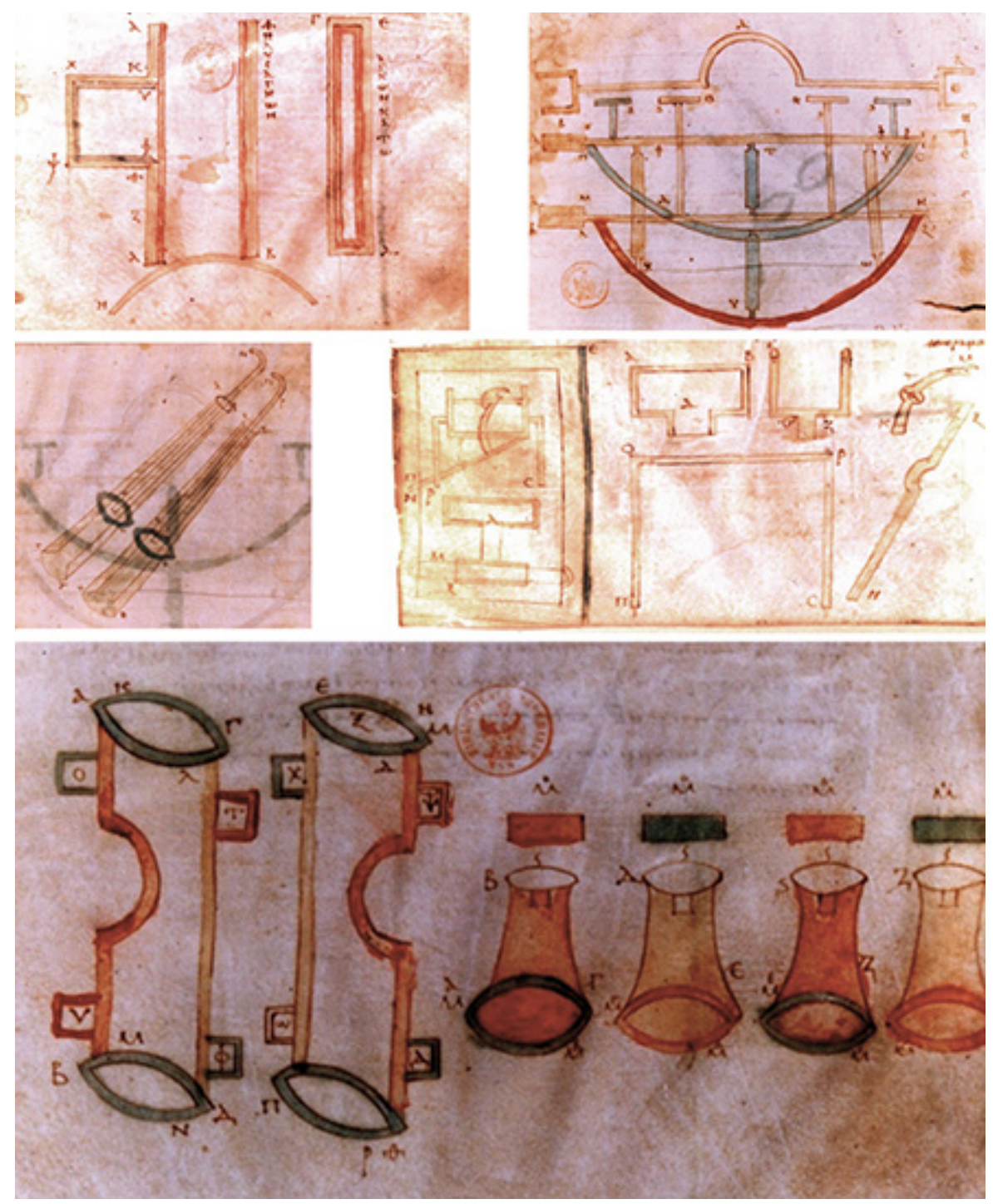
Fig. 4. Corrispondenza tra i componenti della "campestria" (per gentile concessione di Flavio Russo). In basso a sinistra Modulo modiolo. perciò: ma che razza di segreto sarebbe stato alcuni secoli dopo l'ampio impiego di quelle artiglierie su tutti i campi di battaglia ed in tutti gli assedi? E poi, anche a volere ammettere una simile ipotesi, è seriamente credibile che quel veto coinvolgesse tutte le popolazioni dell'età classica? I greci al pari dei fenici e dei punici si astennero dal raffigurarle, e così pure anche i romani e gli egizi, oltre ovviamente ai barbari. Perché tutti ritennero concordemente e per quasi un millennio che quelle immagini non dovessero essere divulgate o rappresentate? Non certo per motivi di pudore rappresentando ben altre scene di guerra e di pace, prive persino dell'infimo livello del buon gusto dell'epoca.

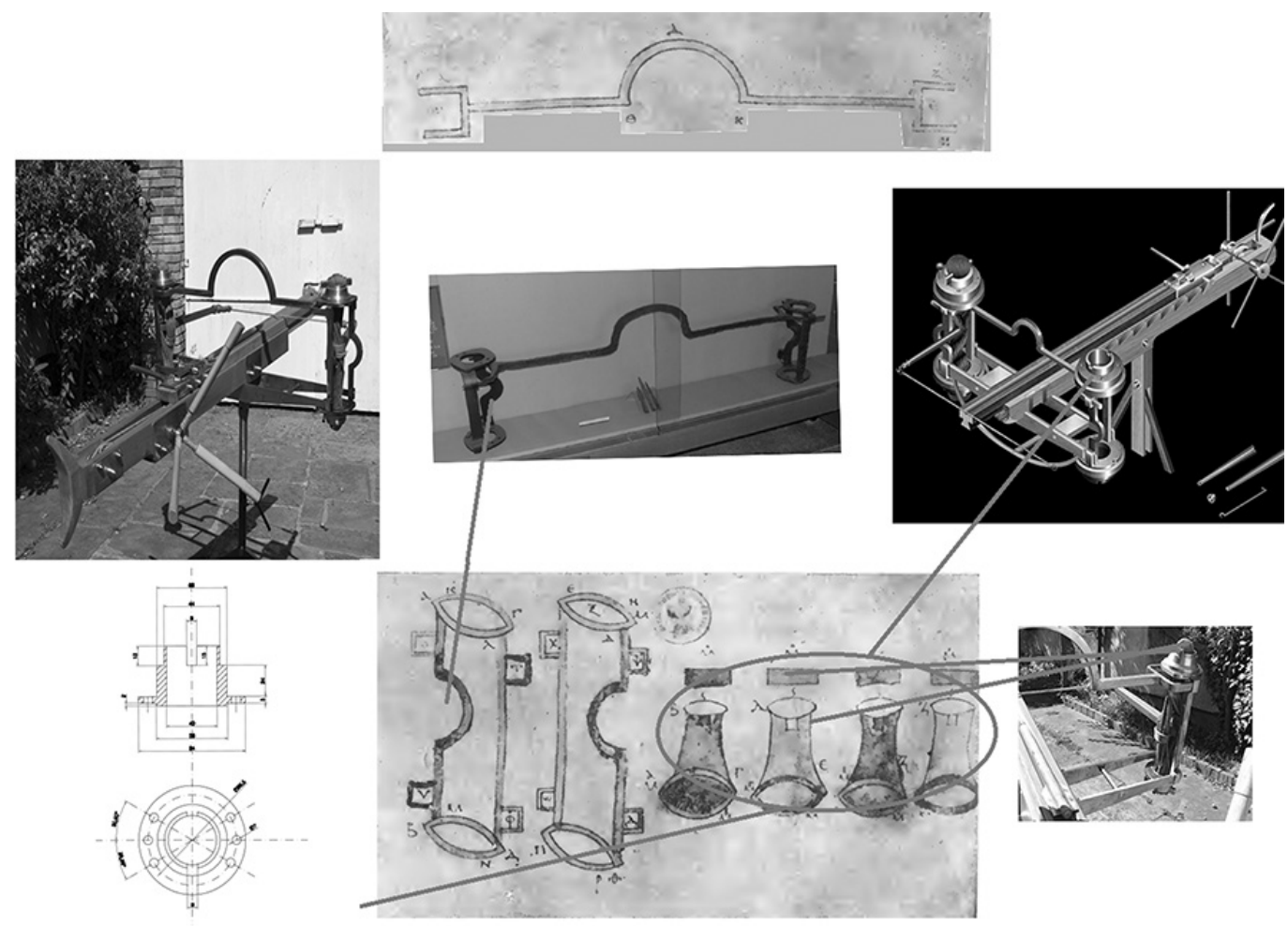

Si potrebbe allora supporre che di tali armi, al di là del lungo arco fruitivo se ne realizzarono ben poche, per cui era difficile conoscerle e farle riconoscere a quanti non le avevano mai viste. In altre parole, assurdo rappresentare oggetti ignoti. Dando per scontato il presupposto, non si può non costatare che anche i centauri nessuno li aveva mai visti, ma non per questo la loro raffigurazione venne evitata! Quanto poi alla supposta inconsistenza numerica, va ricordato che soltanto lungo la cerchia aureliana di Roma nelle 360 torri, anche limitandosi a un unico scorpione per torre, ve ne dovettero essere almeno altrettanti che, tenendo conto della loro longevità non si sbaglia a immaginarne oltre un migliaio avvicendatesi in quell'unica fortificazione nel corso della sua fruizione. Inoltre, tenendo conto di quante città prosperarono tra il IV sec a.C ed il VI, di quante fortezze legionarie vennero erette e di quanti caposaldi insediati lungo i limes, è sensato stimarne l'ammontare a diverse decine di migliaia, prodotte in alcune fabbriche specializzate.

Escluse per quanto accennato le supposizioni precedenti, si deve attribuire tanta laconicità a un'altra motivazione forse meno condivisibile ma non per questo assurda. II morire in guerra, al di là della retorica, non ha mai fatto piacere a nessuno, giovane o meno che fosse: si faceva infatti credere che in quella tragica sorte fosse la grandezza dell'uomo, come nel numero dei figli quella della donna, concetto condiviso soprattutto dai caduti. Per avvalorarlo non si mancava di ricorrere al concetto di virtù guerriera, di sprezzo del pericolo e di valore nei combattimenti col nemico: in altri termini, all'intero repertorio mirante a convincere 
Fig. 5. Ricostruzione digitale dello Scorpione Traiano: LabTAR a.a. I8-19. Esploso Alessandro De Chiara, Andrean Andrea De Stefanis, (ancio della grandezza del morire battendosi con un avversario se non superiore almeno simile ma più fortunato. Quale argomento si sarebbe potuto fornire, però, quando la morte era da un rozzo quadrello di ferro in grado di trapassare elmo e corazza? Quale gloria sarebbe derivata dalla morte inflitta da un dardo scagliato da un vilissimo nemico? Meglio allora non divulgare e soprattutto non esaltare con le immagini, destinate a eternare qualcosa se non di epico almeno di piacevole, macchine di tanto abominio.

Eppure, non per questo quelle armi scomparvero, anzi, furono perfezionate dando origine a nutrite tipologie distinte, non diversamente dalle attuali armi da fuoco, dal fucile al cannone. La mancanza di raffigurazioni, però, ne ha reso improbo riconoscerne i relativi rottami pervenutici: pochissimi, peraltro, tanto da poter essere trasportati in una sola carriola!
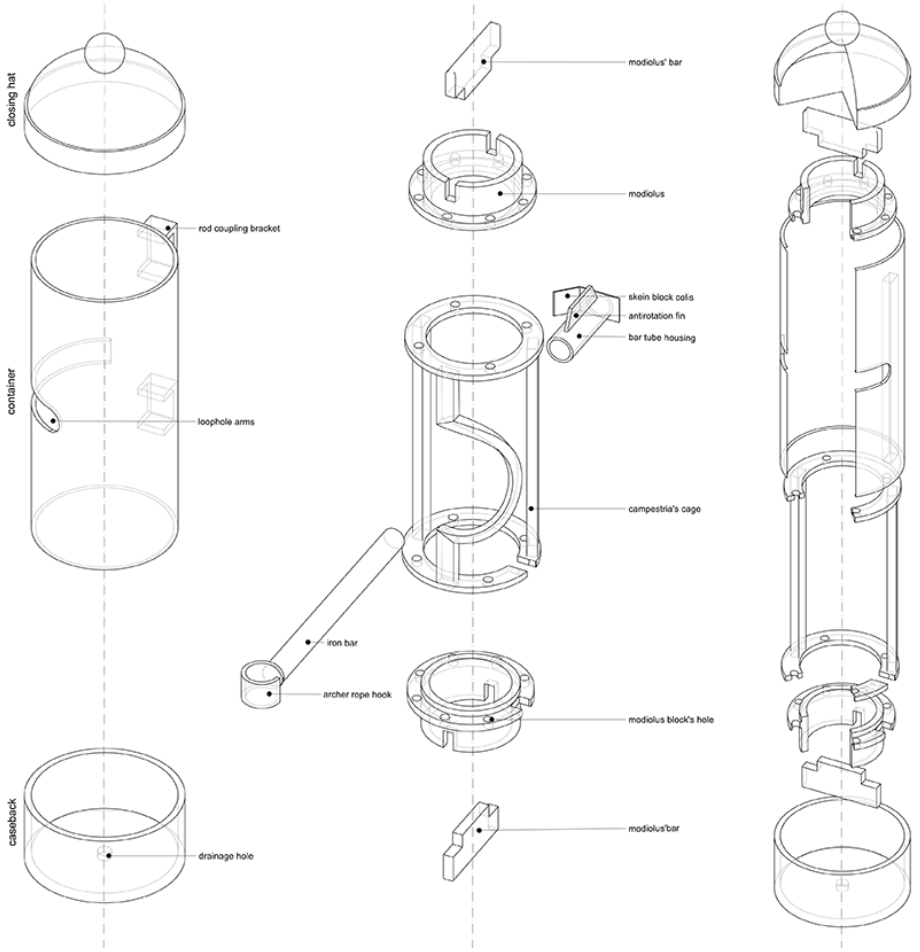

\section{Lo Scorpione Traiano}

Come accennato, la Colonna Traiana (sia pure con parsimonia e per le sole lancia-dardi) sembra finalmente infrangere quella sorta di tabù, innescando tuttavia una vasta disputa interpretativa.

Una prassi innovativa che sembra voler ribadire la ritrovata superiorità nel campo degli armamenti più avanzati e, più in generale, nell'intero comparto delle infrastrutture di supporto tattico, ottenuta con l'apporto dei massimi scienziati, fra i quali Erone di Alessandria (matematico, fisico e ingegnere greco, attivo intorno al II secolo d.C.).

La singolarità di quelle artiglierie - la vera nota distintiva rispetto alle precedenti e di cui l'archeologia ci ha restituito alcuni resti dei congegni propulsori - sono i vistosi cilindri di protezione applicati alle loro matasse elastiche. L'adozione sembra, per molti aspetti, la fase conclusiva di una evoluzione tecnica già ravvisabile, oltre mezzo secolo prima, nei resti di una catapulta della IV Legione Macedonica, rinvenuti a Cremona.

A differenza dei propulsori più antichi, infatti, in questo esemplare compare una protezione frontale posta dinanzi alle due matasse, in modo da ripararle dai frequenti scrosci d'acqua battente, piuttosto che da improbabili offese nemiche (fig. I). 
La spiegazione va ricondotta alla igroscopicità delle fibre nervine, che, se esposte alla pioggia, perdevano del tutto la loro elasticità, privando l'arma della necessaria energia, come avverrà con le artiglierie a fuoco quando si bagnava la polvere pirica!

Le raffigurazioni dello 'scorpione' usato da Traiano nelle campagne daciche, ripetute in più scene lungo le spire della Colonna Traiana, più che chiarire le caratteristiche dell'arma, hanno finito per incrementare le incertezze in merito al loro criterio informatore. Infatti, pur essendo le sole immagini di una macchina da lancio romana tramandata nei diversi allestimenti, non palesano i dettagli del relativo propulsore. Plausibile per una serie di riscontri ritenere che la sua elaborazione sia da ascrivere allo stesso progettista della Colonna, nonché rinomato ingegnere militare al seguito di Traiano, Apollodoro di Damasco (60- I 30 d.C.). Non a caso il grande volume è attribuito - per l'aspetto tecnico - al genio del grande ingegnere Apollodoro di Damasco e - per quello propagandistico - alla scaltrezza dello stesso Traiano. E forse proprio nel ruolo sostenuto dal celebre tecnico sta una plausibile spiegazione delle raffigurazioni delle macchine della colonna traiana.

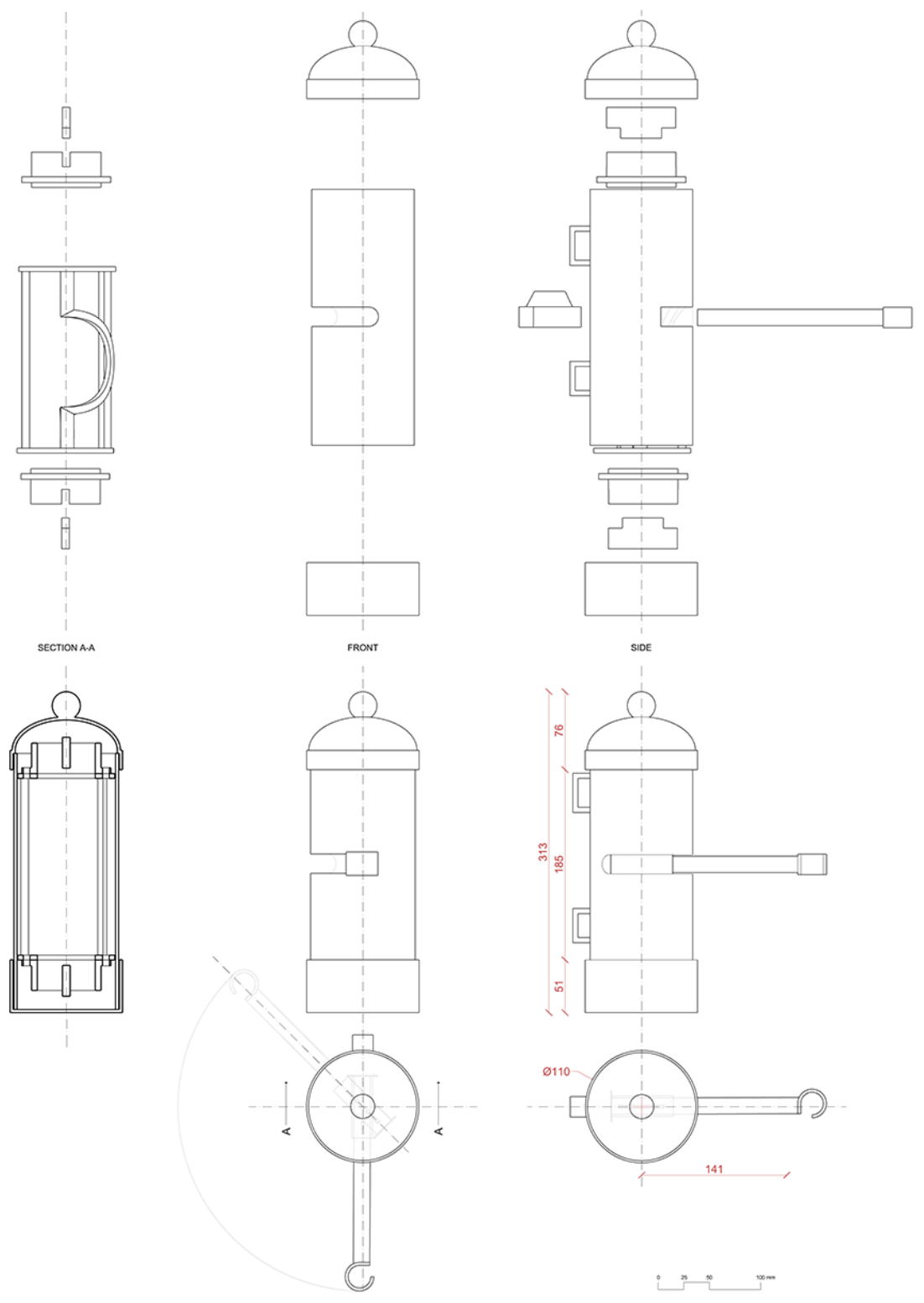


La cheiroballista elaborata da Erone, infatti, conobbe un ulteriore perfezionamento dovuto quasi certamente proprio ad Apollodoro di Damasco che ne protesse le matasse inserendole in appositi cilindri rendendo quel nuovo scorpione idoneo ad operare anche nei teatri nordici, umidi e piovosi e in quanto tali inadeguati ai combattimenti sia in fortezze che soprattutto campali. Nessuna meraviglia perciò che l'autore della colonna abbia così voluto celebrare se stesso come inventore della nuova arma, evidenziandone l'allestimento statico e dinamico, nonché la sua familiarità con Traiano. In definitiva, il progettista a cui andavano ascritti il ponte, la colonna e il nuovo scorpione.

La singolarità di quelle artiglierie, la vera nota distintiva con le precedenti, sono i vistosi cilindri di protezione applicati verosimilmente alle loro matasse elastiche che sembra, per molti aspetti, la fase conclusiva di un progresso innescato dalla forte igroscopicità delle matasse nervine, private di tensione quando bagnate dalla pioggia.
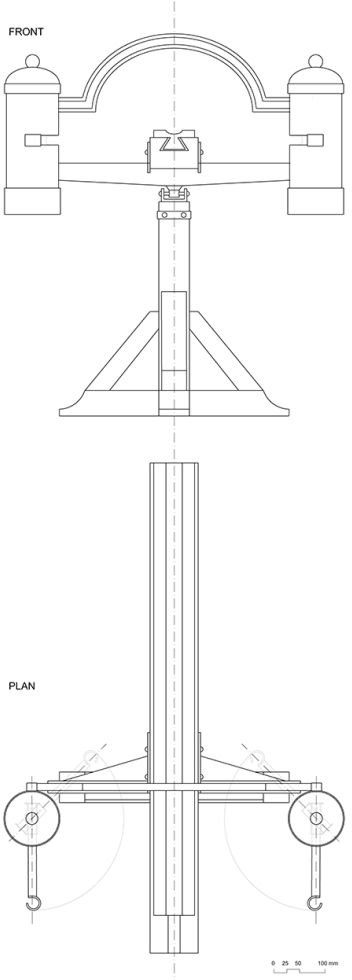

Dal punto di vista militare, poi, quelle immagini che sembrano infrangere l'omertà iconica, in realtà non svelavano nulla, tant'è che anche noi possiamo soltanto ipotizzare il contenuto dei vistosi cilindri che le componevano. Fra quelle istantanee ne spiccano una mezza dozzina che hanno per soggetto un tipo di armamento balistico fino allora ignoto, o non divulgato così palesemente destinato ad avere da quella campagna un ruolo basilare nell'esercito romano. Ad essere esatti sono dei pezzi di artiglieria lanciadardi, scorpioni o forse manubaliste, raffigurate in allestimento da fortezza, campale, ippotrainata leggera e ippotrainata pesante. Anche i pezzi in allestimento ippotrainato sembrano testimoniare l'avvento di un'arma da lancio campale, una lanciadardi destinata a prendere parte attiva nei combattimenti. Armi simili alle precedenti, ma pronte a entrare in azione in qualsiasi momento, persino durante il trasporto, in modo da fornire così una protezione balistica alle colonne in marcia. In un fotogramma almeno, infatti, si scorgono due legionari intenti a porre in punteria una catapulta, mentre i muli la stanno trainando confermandoci la correttezza della conclusione.

Forse fu con queste immagini che si volle notificare la disponibilità di artiglierie leggere in grado di tirare sempre sotto il diluvio o in marcia, contesti che decisero il massacro di Teutoburgo cento anni prima. Altrettanto verosimile che si volle - proprio con quelle immagini 
- infrangere il rigido veto sulla loro rappresentazione, antesignano segreto militare protrattosi per oltre sette secoli, anche perché da esse non si ricavava alcuna informazione sul loro funzionamento! II che ha finito per innescare numerose ipotesi, da allora inseguitesi per tentare di comprendere cosa si nascondesse nei due cilindri che sostituivano la coppia di matasse elastiche della cheiroballistra di Erone, suggestione alla quale non abbiamo neppure noi saputo resistere e forti della logica meccanica e delle precedenti ricostruzioni nel settore, abbiamo provato a sbrogliare l'enigma. Lavoro che resta però solo una nuova ipotesi!

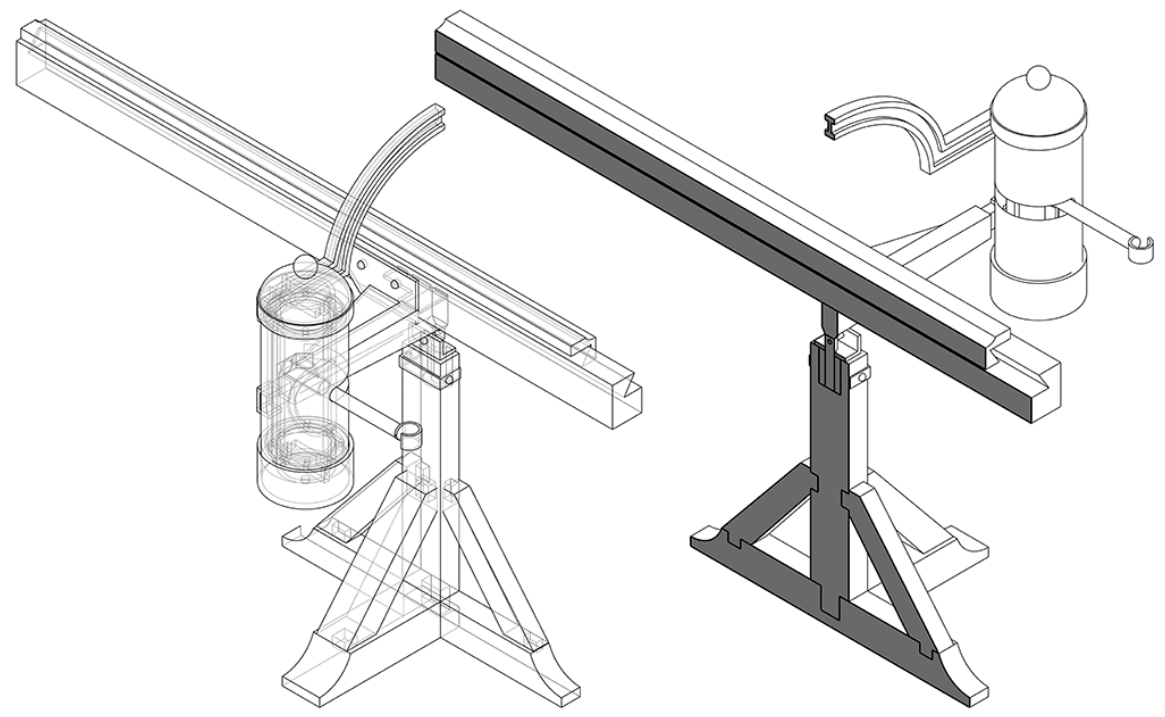

\section{La ricostruzione dello scorpione traiano}

La ricostruzione di antiche macchine belliche deve mostrare coerenza con le regole di produzione, i requisiti tecnologici, nonché con una serie di dettami di natura culturale. Ogni ricostruzione, infatti, deve essere supportata da un preciso riferimento storico-archeologico e attenersi a tre criteri imprescindibili:

- essere documentata nelle fonti scritte;

- disporre di reperti archeologici;

- riferirsi ad almeno una menzione iconografica.

L'efficacia delle attività di archeologia sperimentale è fortemente legata al controllo e alla corretta interpretazione dei reperti archeologici posti alla base delle ricostruzioni. Nondimeno, la progettazione esecutiva, la composizione delle tavole grafiche, la rielaborazione dei dettagli esecutivi e delle viste d'insieme sono da porsi a fondamento dellintero processo operativo. Dalla acquisizione delle fonti alla definizione esecutiva dei componenti, il disegno è il principale strumento di conoscenza delle leggi che governano la struttura formale. II progetto di ricostruzione dello Scorpione Traiano è stato avviato dal gruppo di lavoro Archeotecnica nel 2008. A supporto della progettazione esecutiva, sono state aggregate le attività del Laboratorio di Tecniche Avanzate della Rappresentazione del Dipartimento di Ingegneria dell'Università degli Studi della Campania “Luigi Vanvitelli”. L'attività di ricerca è pedagogicamente ricaduta sull'attività didattica: gli allievi hanno avuto modo di conoscere il funzionamento dell'armamento balistico attraverso l'osservazione dei prototipi costruiti materialmente in scala reale, sulla base dei disegni derivati dalle fonti raccolte (figg. 2, 3, 4). L'assemblaggio, con la necessità concreta di coniugare regole tecniche, di funzionamento e coerenza storica, ha permesso di confermare induttivamente alcuni dei quesiti posti e confrontati con altri studi paralleli. 
Fig. 9. Ricostruzione digitale dello Scorpione Traiano: LabTAR. Viste de modello (rielaborazione di Umberto Palmieri).

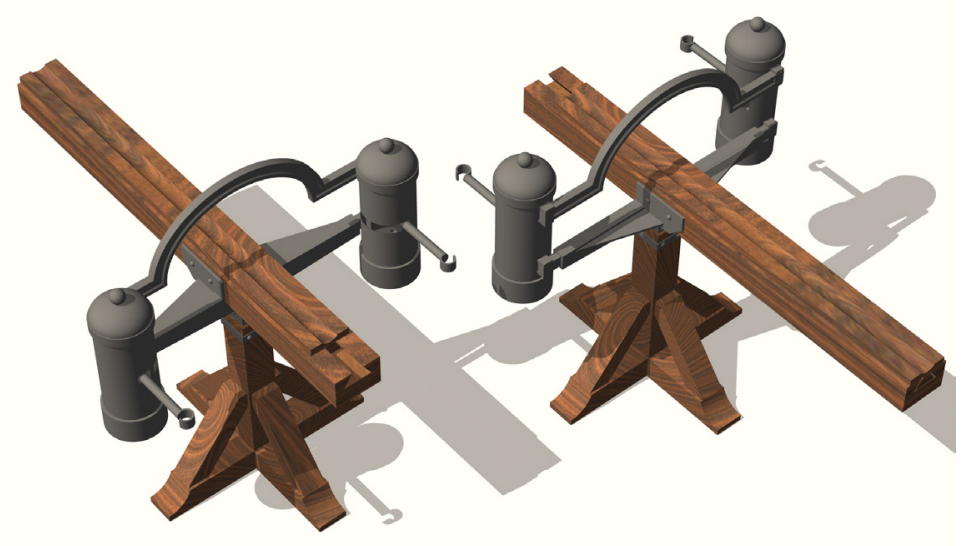

Giova ricordare che molteplici sono le tipologie sottese al termine 'rilievo'. Nell'accezione più ampia, questo importante processo di analisi che precede ogni elaborazione programmatica e idea di modifica dello stato di fatto, si riferisce alla primitiva azione del 'levare', ovvero dell'asportare dal contesto d'interesse quanto di peculiare si coglie nelle sue forme e in funzione degli obiettivi particolari. La procedura, pertanto, è attuata in base all'interesse dell'operatore che si può awalere, per meglio compierla, di adeguati strumenti e precise metodiche (figg. 5- I0).

Fase prodromica è dunque l'acquisizione dei dati che, qualora inerenti all'ambito del rilievo architettonico-topografico, insistono sulla misura di punti nodali. Rispetto a un sistema di riferimento le coordinate levate si ritengono oggettive, essendo dati ripetibili a parità di condizioni iniziali. Ne consegue che il rilevare le misure di un particolare reperto archeologico costituisce la base per la sua conoscenza ma che, tuttavia, necessita di un'accorta interpretazione geometrica per tradursi in opportunità di disegno. La restituzione della forma di un frammento, molto spesso contorto e corroso, non consente di rispondere con sufficiente certezza alle basilari domande circa cosa sia stato, o soprattutto cosa non sia stato; a cosa in concreto servisse; e più ancora come funzionasse. In tali circostanze si rende necessario procedere, come nel caso di un complesso identikit, affidandosi ad una indiscutibile menzione nelle fonti letterarie, un evidente proposizione nelle fonti iconiche (dipinti, bassorilievi, incisioni etc.) nelle quali sia agevolmente identificabile e ai reperti archeologici più o meno similari sicuramente identificati.
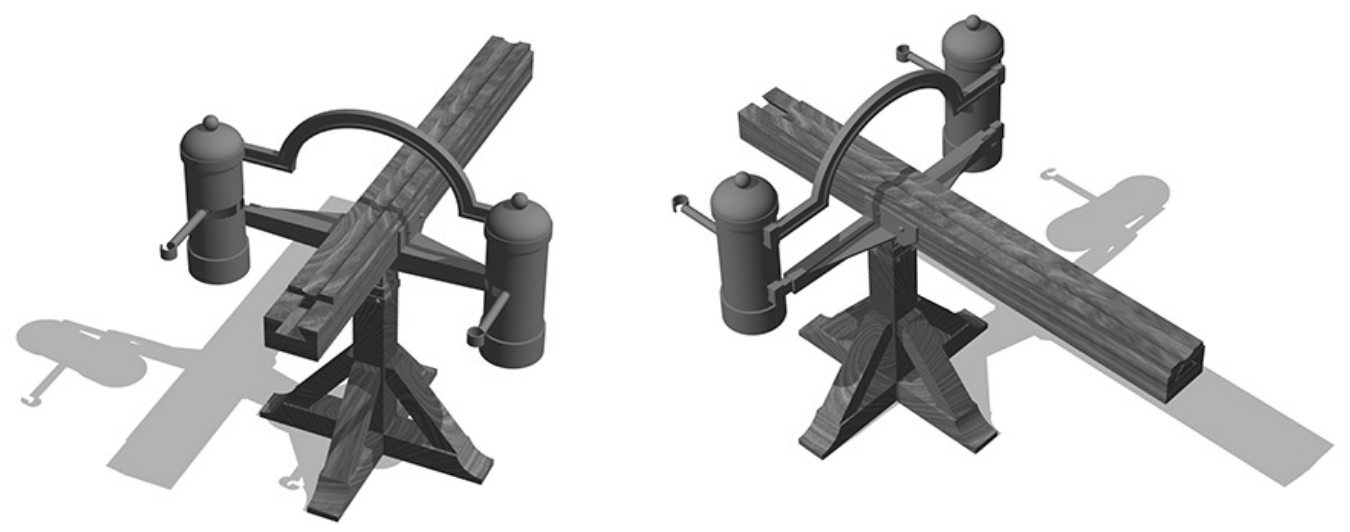
Fig. 1 I. Archotenica Scorpione di Traiano; ricostruzione matricola n. I3/19 Per gentile concessione $d$ Flavio Russo. II dettaglio della campestria.

Fig. 12. Archotenica di Flavio Russo scorpione di Traiano: ricostruzione matricola n.13/19 Per matricola n. 3 / 19 Per gention del motore a torsione.

Fig. 13. Scorpione Traiano: dall'immagine alla rico-
struzione funzionante.
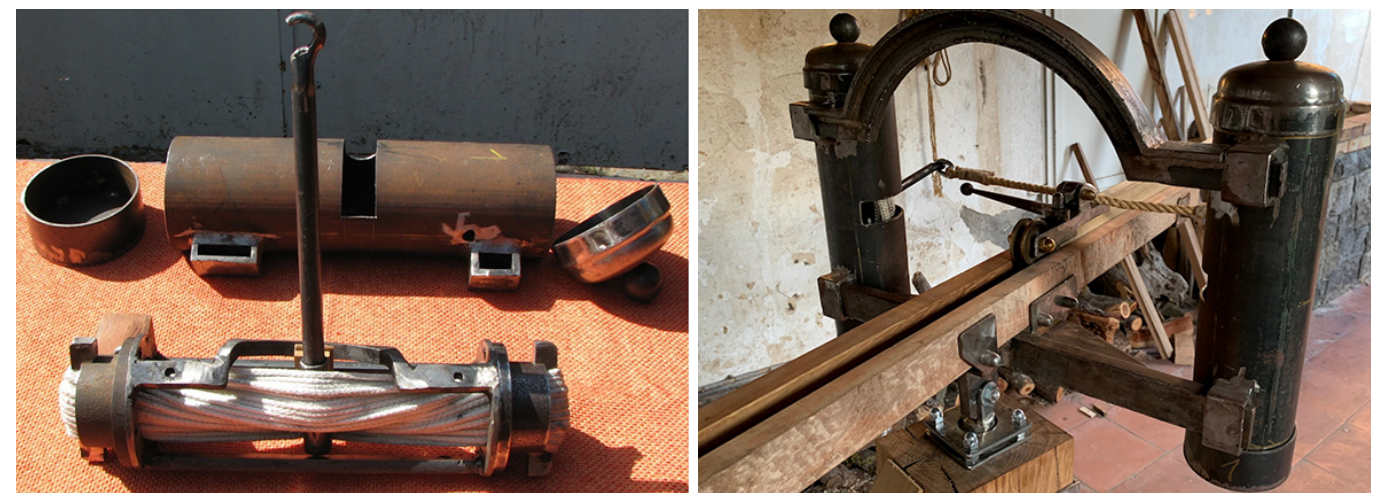

La corretta ricostruzione dell'insieme di appartenenza e di provenienza, deve fondersi, pertanto, nel lavoro di disegno in cui le coeve fonti letterarie, iconiche e materiali, siano parametri di un modello tramite il quale articolare e commutare i dati in un processo che converge nel rilievo critico e progettuale (figg. | | - |3).

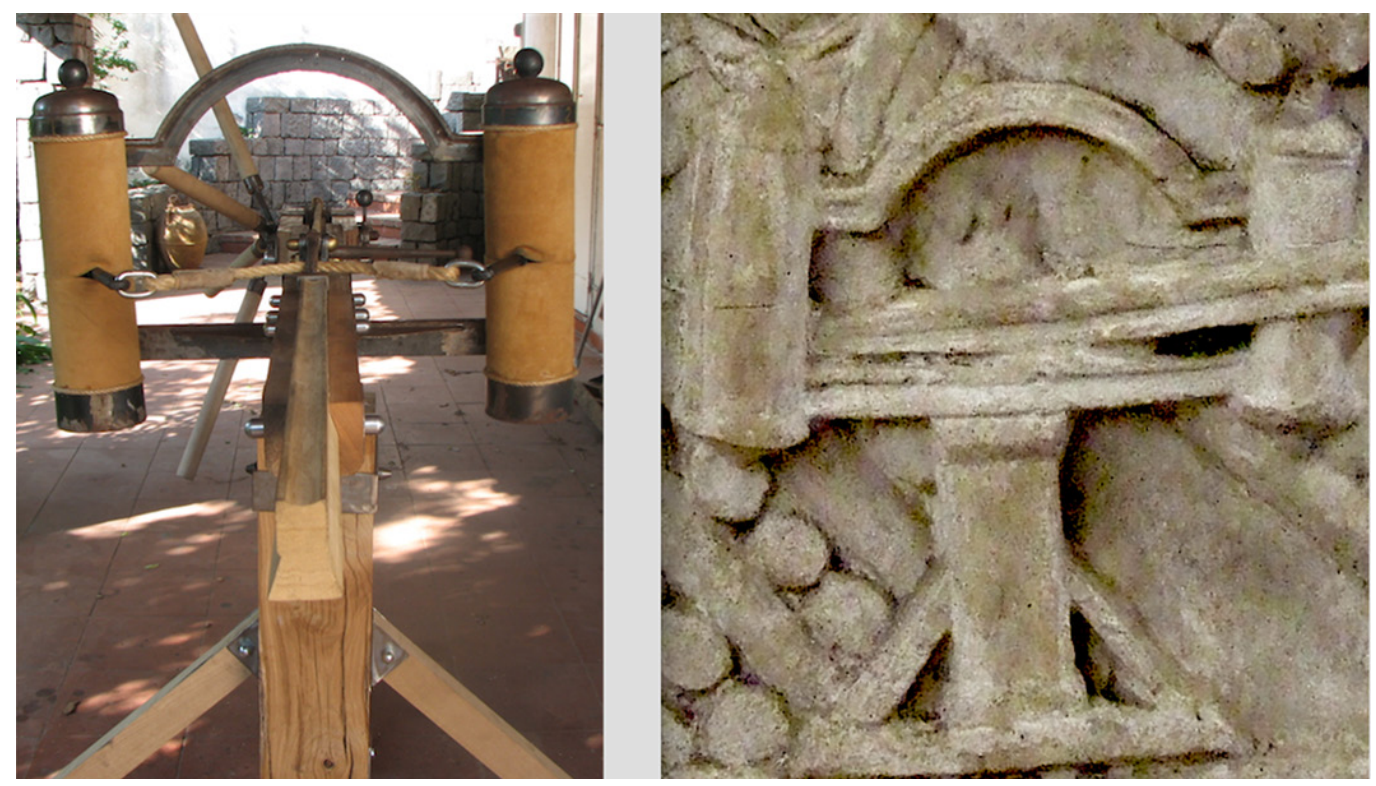

Note

[I] Del gruppo - sorto per volontà e ingegno del dott. Flavio Russo, collaboratore dello stato maggiore dell'esercito - fanno parte, con ruoli e competenze diverse, boscaioli, falegnami, fabbri, scalpellini, cordai che realizzano, oltre agli accessori, le parti grezze delle macchine assemblate con l'aiuto di alcuni collaboratori, motivati e pazienti. Adriana Rossi, professore ordinario di Disegno (Lab.TAR), controlla la corretta modellazione digitale dei reperti archeologici posti alla base dei progetti esecutivi delle ricostruzioni.

[2] Velleio Patercolo, Storia romana (Historiae Romanae ad M. Vinicium libri duo), II, I I7- I20; Cassio Dione Cocceiano, Storia romana, LVI, 19-2I; Publio, Annio Floro, Epitome de T. Livio Bellorum omnium annorum DCC Libri duo, II, 36-37.

[3]Trad. libera: "Racconterai nuovi fiumi scorrenti fra terre, nuovi ponti gettati sui fiumi, eserciti accampati sui dirupi, un re pieno di baldanza cacciato, non che dal trono, dalla vita. Ed oltre a ciò la magnificenza di due trionfi, l'un dei quali fu il primo ottenuto su gente indomita, e l'altro l'ultimo". C. Plinii Caecilii Secundi, Epistolae, VII, 2.

[4] Marsden Eric William (197I). Greek and Roman Artillery. Technical Treatises. Oxford: Clarendon Press, pp. I05- I 84; Marsden Eric William (1969). Greek and Roman Artillery, Historial Development. Oxford: Clarendon; Schramm Erwin (1918). Die Antiken Geschutze der Saalburg. Berlino 1918, rist. 1980 


\section{Riferimenti bibliografici}

Rimandando ad un contesto più appropriato la descrizione in dettaglio del lavoro svolto, si elencano le fonti studiate per eseguire le ricostruzioni digitali di cui quella illustrata è solo un esempio parziale:

Barbaro Daniele ( I567). I dieci libri dell'architettura di M. Vitruvio Tradotti \& commentati da Mons. Daniele Barbaro eletto Patriarca d'Aquileia, da lui riveduti \& ampliati. Venezia: Francesco de' Franceschi Senese. Roma: Bardi, 2006, facsimile.

Barbieri Nicola S. (1984). II primo trattato di tecnologia. La "Costruzione di armi da getto" di Filone di Bisenzio. Introduzione traduzione italiana e commento. Tesi di laurea (non pubblicata) in Lettere e Filosofia, relatore prof. G. A. Ferrari. Università degli Studi di Pavia.

Baatz Dietwulf (1974). Teile spatromischen Ballistenaus Gornea und Orşova (Rumanien). In Saalburg-Jahrbuch, 31 , pp. 50-72.

Baatz Dietwulf (1978). Recent Finds of Ancient Artillery. In Britannia, 9, pp. I- 17

Baatz Dietwulf, Feugere Michel (198I). Elements d'une catapulte romaine trouvee à Lyon. In Gallia, 39, pp. 201-209.

Campbell Duncan B. (2003). Greek and Roman Artillery 399 BC-AD 363. Oxford: Osprey Publishing.

Cesare Cesariano ( 152 I). Di Lucio Vitruvio Pollione De architectura libri dece traducti de latino in vulgare affigurati: commentati: \& con mirando ordine insigniti. Como: Gottardo da Ponte, facsimile. Traduzione di Cesare Cesariano.

Coccejano Cassio Dione (230). Storia romana. Libro LVI. Milano: BUR, I998, pp. I9-2I.

Codex Parisinus inter supplementa Greaca 607 (foll. 56r-58v). Paris: Bibliothèque Nationale, 1867 (Wescher) et 1906 (Schneider).

Coxeter Harold Scott Macdonald,Van de Craats Jan (1993). Philon lines in non-Euclidean planes. In Journal of Geometry, 48, I-2, pp. 26-55.

Diels Hermann, Schramm Erwin (1919). Philons Belopoiika (Abhandlungen der preussischen Akademie der Wissenschaften, Philosoph. hist. KI. 16). Berlin: Reimer.

Diels Hermann, Schramm Erwin (1920). Exzerpte aus Philons Mechanik B. VII und VIII (vulgo funftes Buch) (Abhandlungen der preussischen Akademie der Wissenschaften, Philosoph. hist. KI. 12). Berlin: Reimer.

Drachmann Aage Gerhardt (1948). Ktesibios Philon and Heron: A Study in Ancient Pneumatics. In Acta historica scientiarum naturalium et medicinalium, vol. 4.

Ferrari Gian Arturo (1982). Meccanica allargata. In Giannantoni Gabriele, Vegetti Mario (a cura di) Atti del convegno "La scienza ellenistica". Pavia I4-16 aprile 1982 pp. 227-296. Napoli: Bibliopolis.

Garlan Yvon (1972). La guerre dans l'antiquité. Bologna: II Mulino.

Garlan Yvon (1974). Recherches de poliorcetique grecque. Paris: Ecole francais d'Atenes.

Garlan Yvon (1973). Cités, armées et stratégie à l'époque hellénistique d'après l'oeuvre de Philonde Byzance. In Historia, 22 pp. 16-33.

Guzzomi Andrew Louis, Maraldi Mirko, Molari Pier Gabriele (2012). A historical review of the modulus concept and its relevance to mechanical engineering design today. In Mechanism and MachineTheory, 50, pp. I- I4.

Heronis di Alessandria (o il Vecchio) ( I 572). Liber de machinis bellicis, Venetiis, apud Franciscum Franciscium Senensem.

Landels John Gray ( 878$)$. Engineering in the ancient world. Berkeley: University of California Press.

Landrus Matthew (20 I0). Vinci's Giant Crossbow. Berlin: Springer.

MacDhnouill Gille, Nanzydon the Traveler (1918). A Modern Reconstruction of Vitruvius' Scorpion. Berlin.

Marsden Eric William (1969). Greek and Roman Artillery. Historical Development. Oxford: Clarendon Press.

Marsden Eric William ( 197I). Greek and Roman Artillery. Technical Treatises. Oxford: Clarendon Press, pp. I05- I84.

Molari Pier Gabriele et al. (20 I I). La ricostruzione della balista di Vitruvio. Esercitazioni dell'insegnamento Laboratorio di Storia dell'Ingegneria Meccanica tenuto da P.G. Molari nell' a.a. 20 I0-20I I. Facoltà di Ingegneria di Bologna.

Patercolo Velleio (30 d.C.). Storia romana (Historiae Romanae ad M. Vinicium libri duo), II, pp. I I 7 - I 20

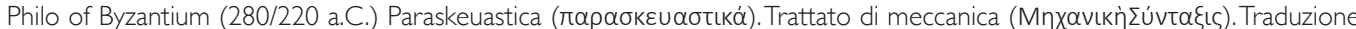
francese di Garlan Yvon (1974). Le livre "V" de la "syntaxe mecanique" de Pihilon de Byzance. In Recherches de poliorcétique grecque. Paris: Ecole Française d'Athenes De Boccard.

Prager Frank David (1974). Philo of Byzantium, Pneumatica: the first treatise on experimental physics, western version and eastern version. Historical Introduction and Technical Commentary by Wiesbaden Schone Richard (ed.) (I 893). Berlin: Philonis Mechanicae Syntaxis, libri quartus et quintus.

Schmidt Wilhelm (ed.). ( 1899). Heronis Alexandrini opera I. Leipzig. pp. 458-89.

Publio Annio Floro (II sec. d.C.). Epitome de T. Livio Bellorum omnium annorum DCC Libri duo, II, 36-37. 
Rance Philip (20I3). Philo of Byzantium. In Roger S. Bagnall et al. (eds.). The Encyclopedia of Ancient History. Wiley-Blackwell; Chichester, E. Sussex/Malden, MA, pp. 5266-5268: <https://onlinelibrary.wiley.com/>

Rossi Adriana (20I5). Executive designs of a palinthytone stone-thrower in reconstructions of ancient Greco-Roman war machines. In <http://www.archeotecnica.com/chi-siamo>.

Rossi Lino (198I). Rotocalchi di pietra. Segni e disegni dei tempi sui monumenti trionfali dell'Impero Romano. Milano: Jaca Book.

Rossi Cesare, Russo Flavio, Russo Ferruccio (2009). Ancient engineers' inventions. Precursors of the present. Berlin: Springer.

Russo Flavio (2002). Tormenta, venti secoli di artiglieria meccanica. Roma: Stato Maggiore Esercito.

Russo Flavio (2004). La catapulta di Vitruvio. In Russo Flavio. L'artiglieria delle legioni romane. Roma: Libreria dello Stato, pp. I99. 280.

Schramm Erwin (1980). Die Antiken Geschutze der Saalburg. Berlino: Homburg.

Schone Richard (ed.) (1893). Philonis Mechanicae Syntaxis, libri quartus et quintus. Berlin.

Drachmann Aage Gerhardt (1948). Ktesibios, Philon and Heron. A study in ancient pneumatics. Copenhagen: E. Munksgaard.

Soedel Werner, Foley Vernard (1979). Ancient Catapults. In Scientific American, 3, 240, pp. I 50 - I 6 I.

Solís Santos Carlos (1996). Macchine, tecniche e meccanica. In Salvatore Settis (a cura di). Storia dei Greci e dei Romani, vol. 7 , pp. 723-24.

Taccola Mariano (I 38 | - 453 circa). De Ingeneis. vol. 2. Wiesbaden: Reichert Verlag. Facsimile.

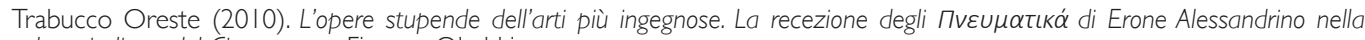
cultura italiana del Cinquecento. Firenze: Olschki.

Valturio Roberto ( 1472). De re militari. Biblioteca civica di Verona, inc.

Vitruvio Marco Pollione (1990). De Architectura - Libri X.Traduzione di Migotto Luciano. Pordenone: Ed. Studio Tesi.

Vitruvio Marco Pollione (2002). De Architectura - Libri X.Traduzione di Bossalino Franca. Roma: Edizioni Kappa.

Vitucci Giovanni (a cura di). (1974). La guerra giudaica. Milano: Mondadori.

Wescher Carl (20 I0). Poliorectique Des Grecs. Traites Theoriques, Recits Historiques. Whitefish: Kessinger Publishing.

Whitehead David (ed.) (1990). Aineias the Tactician. How to Survive Under Siege. Oxford: Clarendon Press.

Wilkins Alan (1995). Reconstructing cheirobbalista. In Journal of Roman Military Equipment Studies, 6, pp. 5-59.

Wilkins Alan (2003). Roman Artillery. Princes Risborough: Shire Publications.

Wilkins Alan, Morgan Len (2000). Scorpio and Cheiroballistra. In Journal of Roman Military Equipment Studies, I I, pp. 77- I 0 I.

\section{Autori}

Adriana Rossi, Università degli Studi della Campania “Luigi Vanvitelli”, adriana.rossi@unicampania.it

Umberto Palmieri, Università degli Studi della Campania “Luigi Vanvitelli”, umberto.palmieri@unicampania.it

Per citare questo capitolo: Rossi Adriana, Palmieri Umberto (2020). Le immagini negate/The denied images. In Arena A., Arena M., Brandolino R.G., Colistra D., Ginex G., Mediati D., Nucifora S., Raffa P. (a cura di). Connettere. Un disegno per annodare e tessere. Atti del $42^{\circ}$ Convegno Internazionale dei Docenti delle Discipline della Rappresentazione/Connecting. Drawing for weaving relationships. Proceedings of the 42th International Conference of Representation Disciplines Teachers. Milano: FrancoAngeli, pp. 803-828. 


\title{
The Denied Images
}

\author{
Adriana Rossi \\ Umberto Palmieri
}

Abstract

Within the aims proposed by the laboratory of real working models (see www.archeotecnica.com) [I] it was intended to promote and enhance the research of engineers, architects and designers experts in survey and representation (ICAR/I7), machine design (ING-IND/I 4 and I5), technology (ICAR I2) and museum settings (ICAR/I4) in the diachronic awareness of events and historical evolutions (ICAR/ I8). In all the above-mentioned fields communication has always used the language of technical design. The evolution of hardware and software has made it possible, then, to extend the traditional meaning of 'drawing' to that of 'model' whose characteristics, thanks to advanced CAD, go beyond the geometrical form to understand all the information aspects that can anticipate the construction of construction sites with a level of control and verification previously unthinkable. Here we present some applied research.

Keywords

survey, exhibit design, stone-thrower, heritage, sustainable tourism.

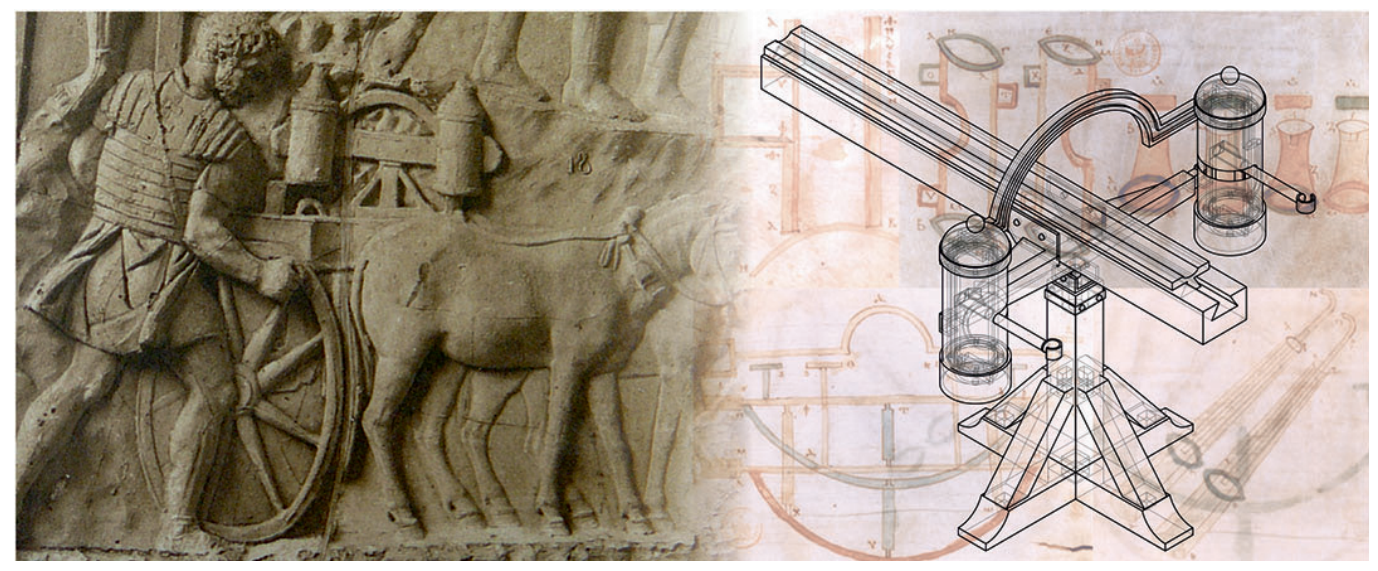




\section{Introduction}

It was probably a coincidence or, more likely, a specific circumstance, since even the Romans did not miss the fatal occasions. Exactly one hundred years after the defeat of Teutoburg (9 A.D.) [2], the most conspicuous celebratory monument of imperial production was designed: the Trajan Column. Inaugurated in I13, it exalted the army and its supreme leader; along its coils there are 150 'shots' inherent in the salient episodes of the war against Dacia (101-106), a suitable background for the emphasis on the most advanced technical-military capabilities of a superior civilization, as Pliny the Younger had just mentioned in a letter: "Dices immissa terris nova flumina, novos pontes fluminibus iniectos, insessa castris montium abrupta, pulsum regia pulsum etiam vita regem nihil desperantem; super haec actos bis triumphos, quorum alter ex invicta gente primus, alter novissimus fuit" [C. Plinii Caecilii Secundi Epistolae, VII, 2] [3].

Among those many 'frames' stand out a dozen that have as subject a type of ballistic weaponry hitherto unknown, or not disclosed so blatantly, destined to have, from that moment on, a basic role in the Roman army. They are pieces of light artillery or darts launcher, properly called 'catapults' and vulgarly 'scorpions', reproduced in all the different settings: from fortress, field, light hippotrained and heavy hippotrained [Russo 2002, pp. 232-243].

Although the present age can, with good reason, be defined as 'of images', this is not the reason why the previous ones were not. In particular, the classical one, has handed down to us an innumerable and extremely varied quantity, able to provide a basic contribution to the knowledge of the past, perhaps the most objective and reliable. It is obvious, therefore, to equate those iconic sources with the traditional ones: every aspect of life, every remarkable real or mythological event, every significant personage deserved, in fact, some representation, often on a particular support. Just to give an example: the coins, of wide diffusion and considerable solidity, were considered ideal to receive the portraits of the powerful or the

Fig. I. Fragment of the Trajan Column. Forum of Rome, 2nd century ( 110 1 I3). War against the Dacians. In the foreground, the Scorpion attributed to the genius of Apollodorus of Damascus (50/60-130). Identification of the modulus-modiolus. Sizing refers to the measures dictated by the measures dictated by Cheiroballista, 62 d.C.

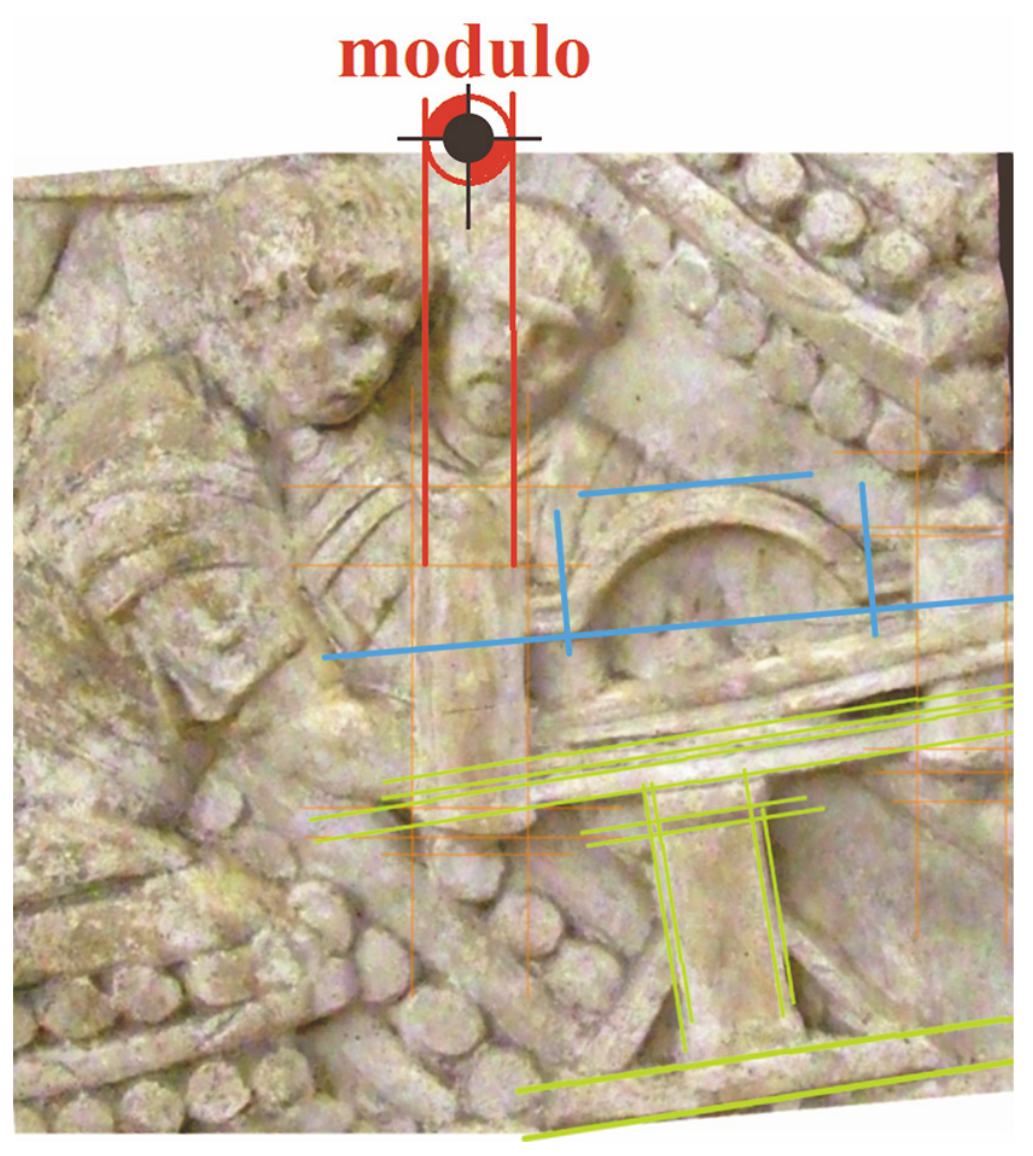


emblems of power; the walls of the houses, of vast surface and well sheltered from the weather, were so for frescoes of landscapes or mythological subjects; the marble bas-reliefs, insensitive to the outrages of the time, were chosen for celebratory re-enactments; stone and bronze statues for the depiction of human or divine characters to be proposed to the admiration or cult of posterity; jewels and gems for engravings and scaramantic, allegorical or erotic figurines. It makes sense to conclude that our ancestors have not neglected anything of their existence, handing down every aspect of their existence to us.

Next to reality, as said, fantastic, mythological, legendary images abound: an orgy of divinities, centaurs, chimeras, satyrs and monsters of various kinds populate those iconic narratives. There are also epic scenes with land and naval battles, sieges and massacres. Whatever the sources write, it becomes possible for us - with a little patience- to find the corresponding image. A precision that seems to be stringent, especially in the field of technology, craftsmanship and construction in general, since they are by their very nature the true novelties of the planet and culture.

Fig. 2. 'Archotenica' by Flavio Russo. Visit to the workshops a.a. 16-17. LabTAR (Advanced Representation Techniques Product prof. Adriana Rossi. LMI2 Engineering Dept., LuigiVanvitelli).
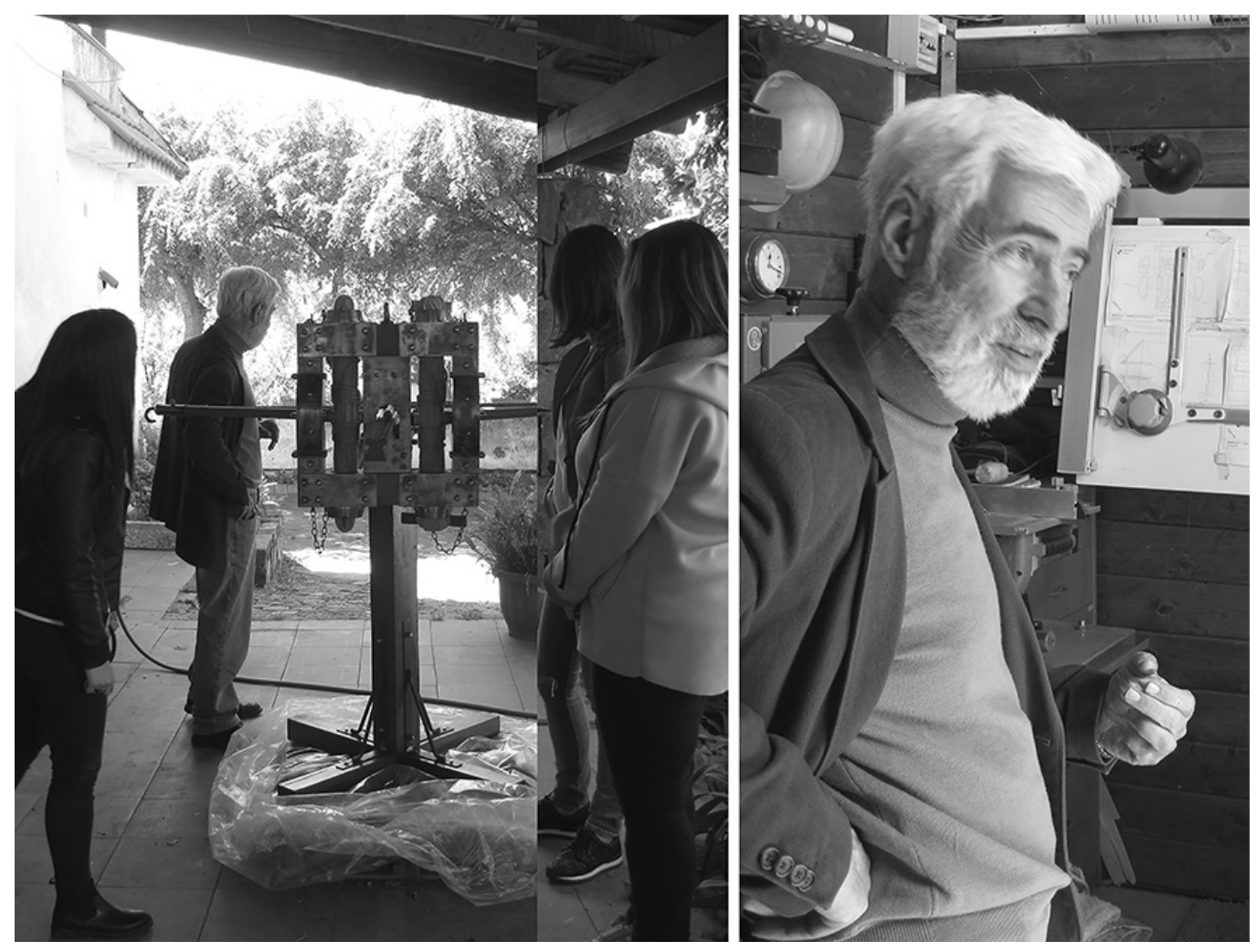

\section{Ballistic weaponry}

It is certainly significant that the Romans defined the machines used in sieges to break the enemy's defense: in practice the wide range of devices to climb over the walls or to break down their defenders from a considerable distance. Later, with the advent of the torsion machines, they renamed the tormenta launch machines. Machines always of considerable complexity to which, however, perhaps because of the contempt that surrounded the manual work of slaves, the contemporaries dedicated very few descriptions and even less images [Russo 2004, pp. 199-280].

Aristotle, giving yet another proof of his refined reasoning, contesting Plato's philosophical lucubrations, affirmed that war should avail itself not only of physical virtue but also of te- 
chnical virtue, strength and ingenuity, making an explicit reference to artillery, of which he recalls the precision and power now achieved. Paradoxically, both in the short period before and after, over a millennium, very few felt the need to provide a detailed description and, less than ever, a representation of those weapons.

Machines of which, in fact, we have no explicit image, to the point of even doubting their concrete existence. To tell the truth, we have partial ones, limited to a detail of their propeller: to be exact, we have only two in over two centuries of use! [4].

How to say two uncertain partial representations of the engine of a car, when millions of them have been circulating for more than a century! Why did the ancients conspire to that almost total and absolute silence? Why did they not want to hand down to us those mechanical conquests of theirs? Why did they never feel proud to portray them in any way? And when they finally painted some of them on the Trajan Column, their two-cylinder engine remained so enigmatic that it suggested the most absurd lucubrations: how to place powerful coil springs in a $16 \mathrm{~mm}$ steel square, inside the same cylinders? Too bad the Romans didn't know either coil springs or solid steel!

The first explanation that comes to mind, insists on an exquisitely military practice: keeping jealously secret every weapon of revolutionary war consequentiality. Military secret, therefore: but what kind of secret would it be a few centuries after the extensive use of those
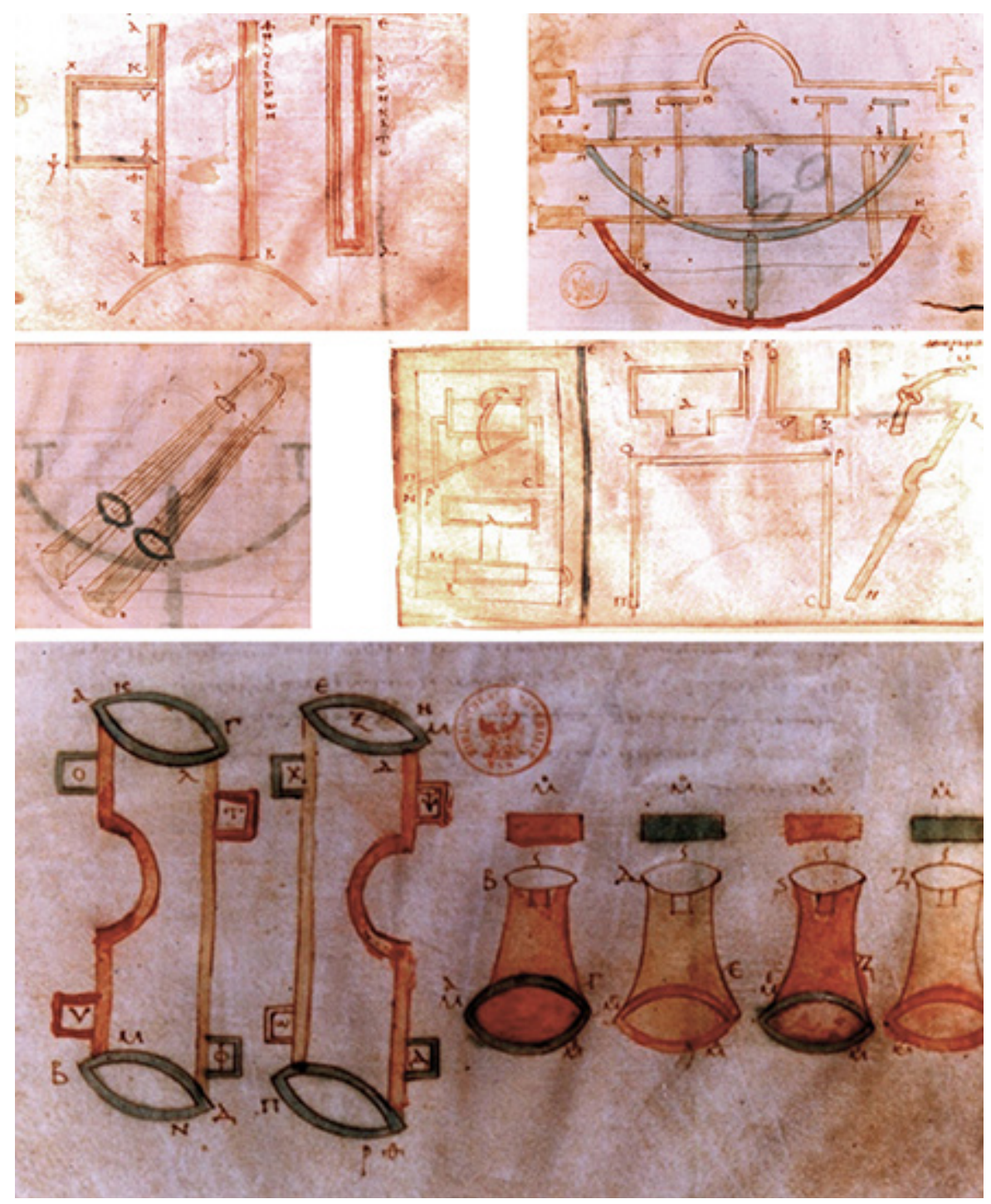
artillery on all battlefields and in all sieges? And then, even to admit such a hypothesis, is it seriously credible that that veto involved all the populations of the classical age? The Greeks, like the Phoenicians and the Punic, refrained from depicting them, as did the Romans and the Egyptians, as well as the barbarians, of course. Why did everyone agree for almost a millennium that these images should not be divulged or represented? Certainly not for reasons of modesty, since they represented many other scenes of war and peace, lacking even the lowest level of good taste of the time.

Fig. 4. Correspondence between the members of 'campestria'. Courtesy of 'Archeotecnica' by Flavio Russo. Lower left Modulo modiolo.

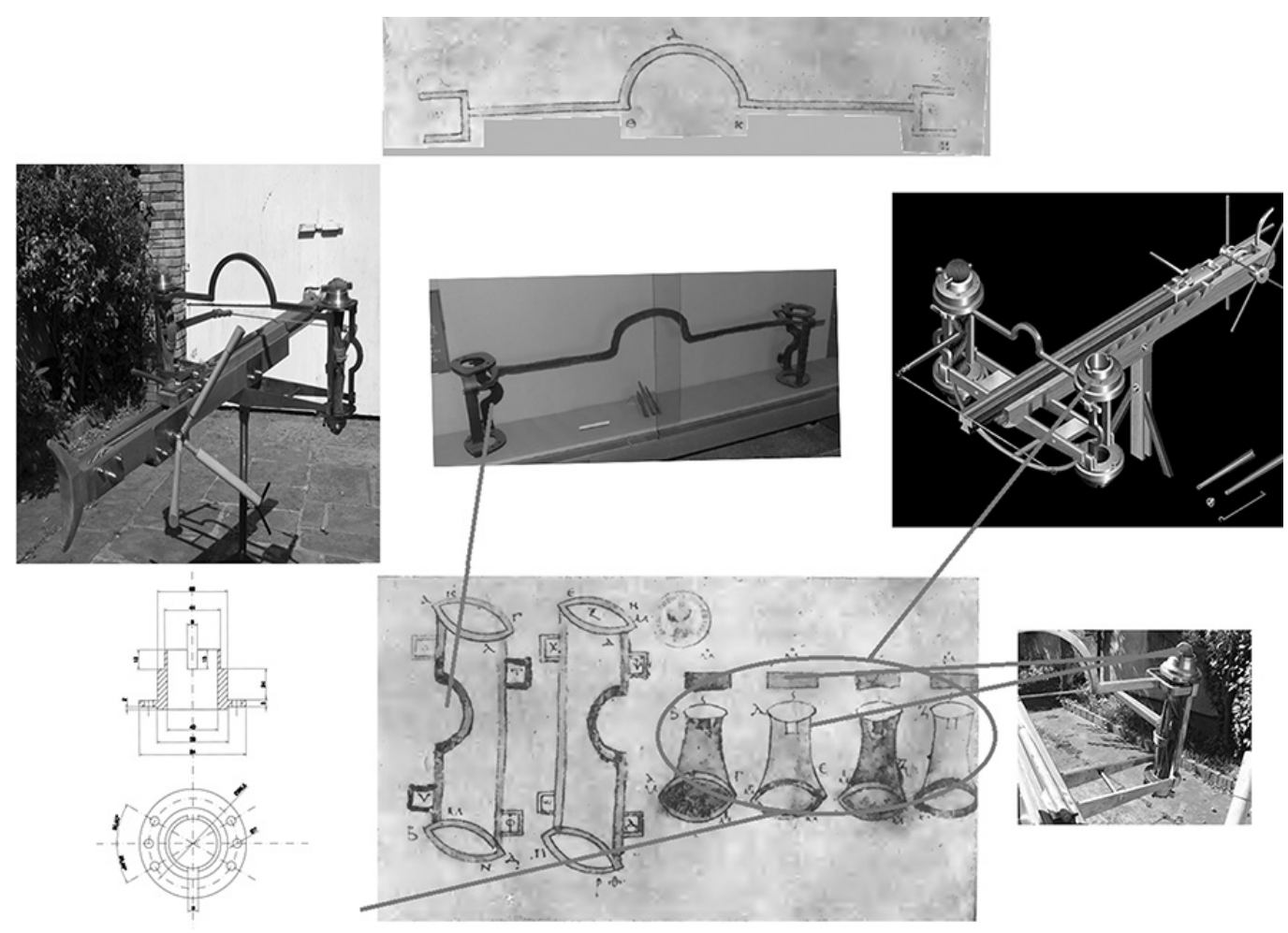

One could then suppose that very few of these weapons, beyond the long fruition period, were made, so it was difficult to know them and make them known to those who had never seen them. In other words, it was absurd to represent unknown objects. Taking the assumption for granted, one can't help but notice that even the centaurs had never seen them, but not for this reason their representation was avoided! As for the supposed numerical inconsistency, it should be remembered that only along the Aurelian circle of Rome in the 360 towers, even if limited to a single scorpion per tower, there had to be at least as many that, taking into account their longevity is not wrong to imagine more than a thousand in that one fortification during its use. Moreover, taking into account how many cities prospered between the 4th century BC and the 6th century BC, how many legionary fortresses were erected and how many strongholds settled along the limes, it makes sense to estimate the amount at several tens of thousands, produced in some specialized factories.

Excluding what has been mentioned above, we must attribute so much laconicity to another motivation, perhaps less acceptable but not for this reason absurd. Dying in war, beyond the rhetoric, has never pleased anyone, young or not: in fact, it was believed that in that tragic fate was the greatness of the man, as in the number of children that of the woman, a concept shared especially by the fallen. In order to confirm this, the concept of warrior virtue, contempt for danger and valour in combat with the enemy was not lacking: in other words, the entire repertoire aimed at convincing people of the greatness of dying by fighting with 
an adversary, if not superior, at least similar but luckier. What argument could have been given, however, when death was from a crude iron square able to pass through helmet and armour? What glory would have derived from death inflicted by a dart thrown by a vile enemy? It is better then not to divulge and above all not to exalt with images, destined to eternalize something if not epic at least of pleasure, machines of such abomination.

And yet, not for this reason those weapons disappeared, on the contrary, they were perfected giving rise to distinct types, not unlike the current firearms, from rifle to cannon. The lack of depictions, however, made it difficult to recognize the relative wreckage: very few, however, so much so that they could be transported in a single wheelbarrow!
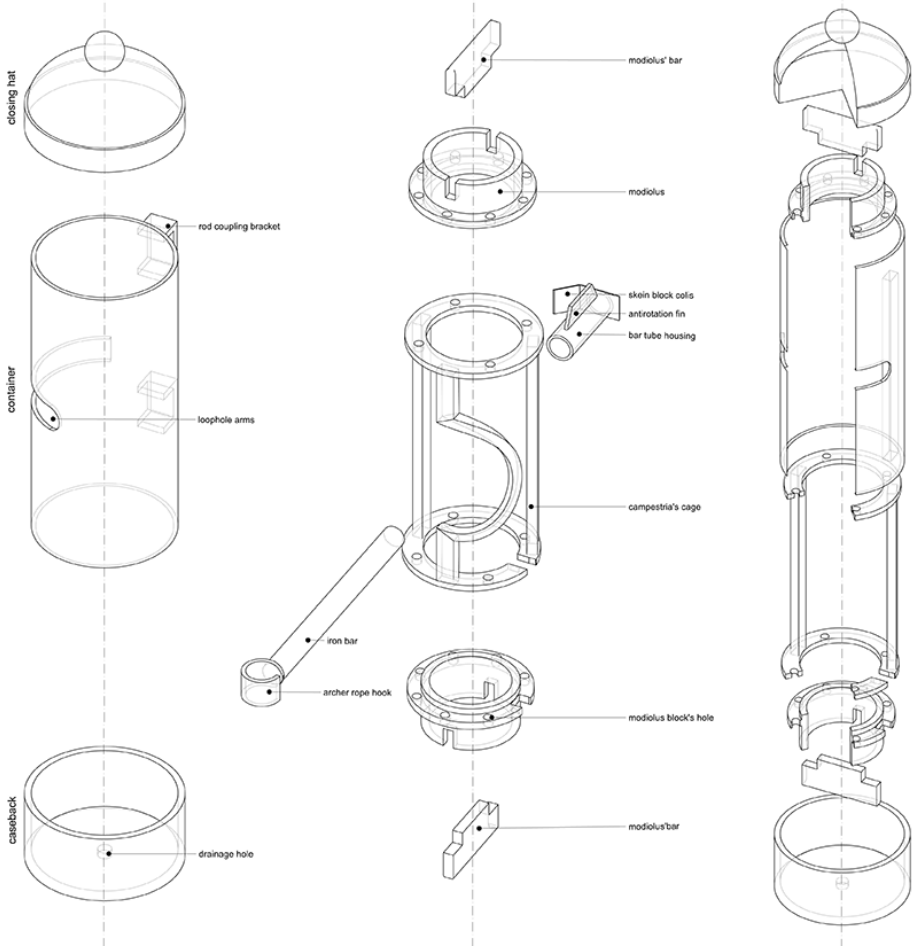

\section{The Trajan Scorpion}

As mentioned, the Trajan Column finally seems to break (albeit sparingly and for dartthrowers only) that sort of taboo yet triggering a vast interpretative dispute.

An innovative praxis that seems to want to reaffirm the newfound superiority in the field of the most advanced armaments and, more generally, in the entire sector of tactical support infrastructures, obtained with the contribution of the greatest scientists, including Hero of Alexandria (Greek mathematician, physicist and engineer, active around the second century AD).

The singularity of those artilleries -the real distinguishing feature compared to the previous ones and of which archaeology has given us some remains of the propulsion devices- are the showy protection cylinders applied to their elastic hanks. The adoption, in many respects, seems to be the final phase of a technical evolution already recognizable, more than half a century earlier, in the remains of a catapult of the IV Macedonian Legion, found in Cremona. Unlike the older engines, in fact, in this exemplar there is a frontal protection placed in front of the two skeins, in order to protect them from driving rain, rather than from improbable enemy offenses (fig. I). The explanation can be traced back to the hygroscopicity of the 
nerve fibers, which, when exposed to rain, completely lost their elasticity, depriving the weapon of the necessary energy, as will happen with artillery fire when the pyric dust got wet! The depictions of the "scorpion" used by Trajan in the Dacian campaigns, repeated in several scenes along the coils of the Trajan Column, rather than clarifying the characteristics of the weapon, have ended up increasing the uncertainties regarding their informant criterion. In fact, although they are the only images of a Roman launch machine handed down in the various settings, they do not reveal the details of its engine. It is plausible for a series of findings to believe that its elaboration is attributable to the same designer of the Column, as well as renowned military engineer in the wake of Trajan, Apollodorus of Damascus (60- I 30 A.D.). It is no coincidence that the great volume is attributed -for the technical aspect- to the genius of the great engineer Apollodorus of Damascus and -for the propaganda aspect- to the cunning of Trajan himself. And perhaps it is precisely in the role supported by the famous technician that there is a plausible explanation of the representations of the machines of the Trajan column.

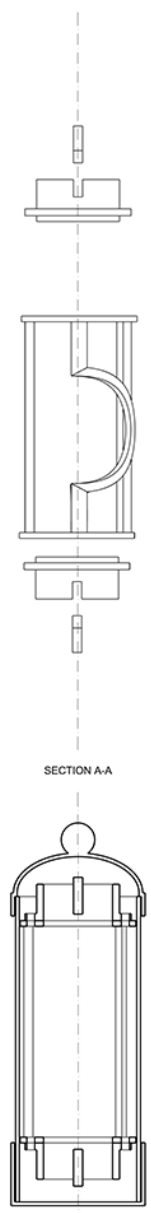

ig. 6. Digital reconstruction of Trajan Scorpio: LabTAR a.a. I8-19. Orthogonal projections of campestria (students: Alessandro De Chiara, Andrea De Stefanis, Umberto Palmieri).
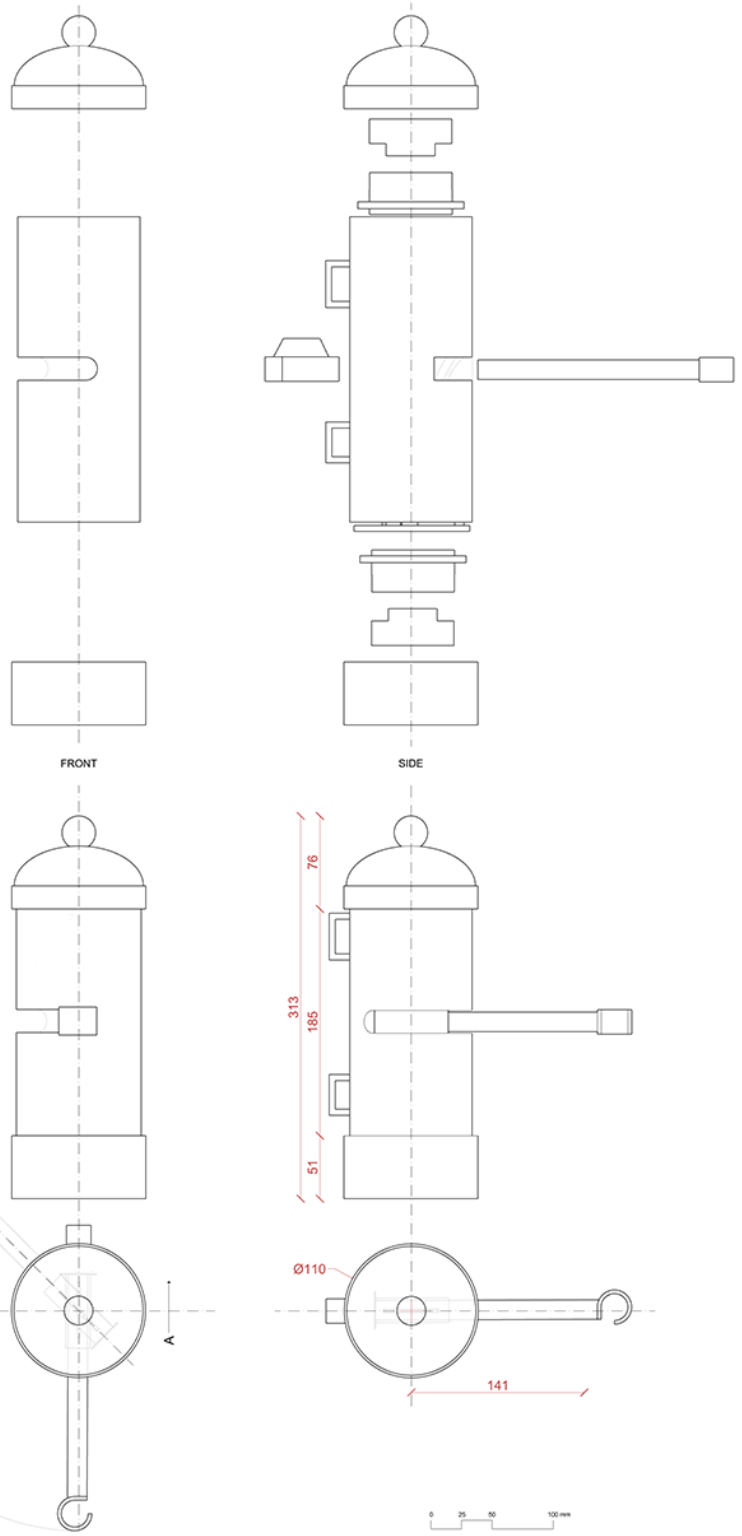
Fig. 7. Digital reconstruction of Trajan Scorpio: LabTAR a.a. 17-18. Plan and elevation of the whole. (students: Rosa Anzalone Federeica Damasco, Maria Grieco).
The cheiroballistra elaborated by Hero, in fact, knew a further improvement due almost certainly to Apollodoro of Damascus who protected the skeins by inserting them in special cylinders making that new scorpion suitable to operate even in the Nordic theaters, wet and rainy and as such unsuitable for fighting both in fortresses and especially in the field. No wonder, therefore, that the author of the column wanted to celebrate himself as the inventor of the new weapon, highlighting its static and dynamic layout, as well as his familiarity with Trajan. In short, the designer to whom the bridge, the column and the new scorpion were attributed.

The singularity of those artillery, the real distinctive note with the previous ones, are the showy protection cylinders probably applied to their elastic skeins that seem, in many ways, the final phase of a progress triggered by the strong hygroscopicity of the nerve skeins, deprived of tension when wet by rain.

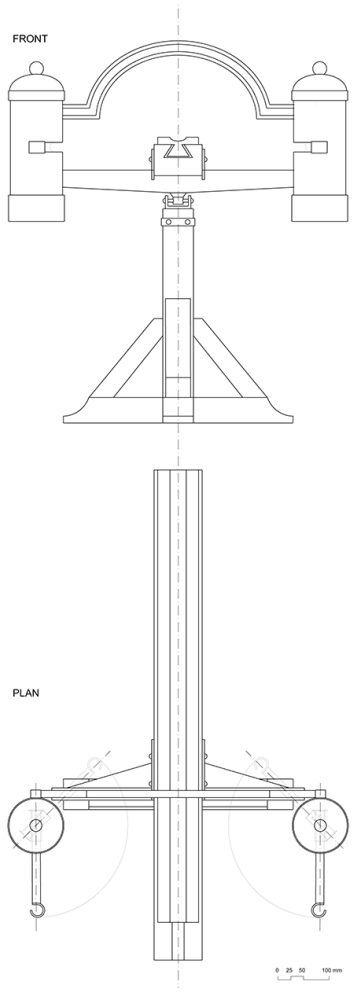

From the military point of view, then, those images that seem to break the iconic omertà, in reality, did not reveal anything, so much so that even we can only hypothesize the content of the showy cylinders that composed them. Among those snapshots there are half a dozen that have as subject a type of ballistic weaponry so far unknown, or not disclosed so obviously destined to have from that campaign a basic role in the Roman army. To be exact, they are artillery pieces of spear throwers, scorpions or perhaps manuballista, depicted in fortress, campal, light hippotrainata and heavy hippotrainata. The hippotrained pieces also seem to bear witness to the advent of a field throwing weapon, a spear thrower destined to take an active part in combat. Weapons similar to the previous ones, but ready to enter into action at any time, even during transport, so as to provide ballistic protection to the marching columns. In at least one frame, in fact, we can see two legionnaires intent on placing a catapult in the infantry, while the mules are towing it, confirming the correctness of the conclusion.

Perhaps it was with these images that they wanted to notify the availability of light artillery capable of pulling always under the flood or on the march, contexts that decided the massacre of Teutoburg one hundred years earlier. It is equally probable that it was with these 
Fig. 8. Digital reconstruction of Trajan Scorpio: axonometric views (graphic elaboration by Umberto Palmieri). images that they wanted to break the strict veto on their representation, a secret military forerunner for over seven centuries, also because no information on their functioning could be obtained from them! This ended up triggering numerous hypotheses, since then pursued to try to understand what was hidden in the two cylinders that replaced the pair of elastic skeins of Hero's cheiroballistra, a suggestion to which not even we have been able to resist and strong of mechanical logic and previous reconstructions in the sector, we tried to unravel the enigma. Work that remains, however, only a new hypothesis!

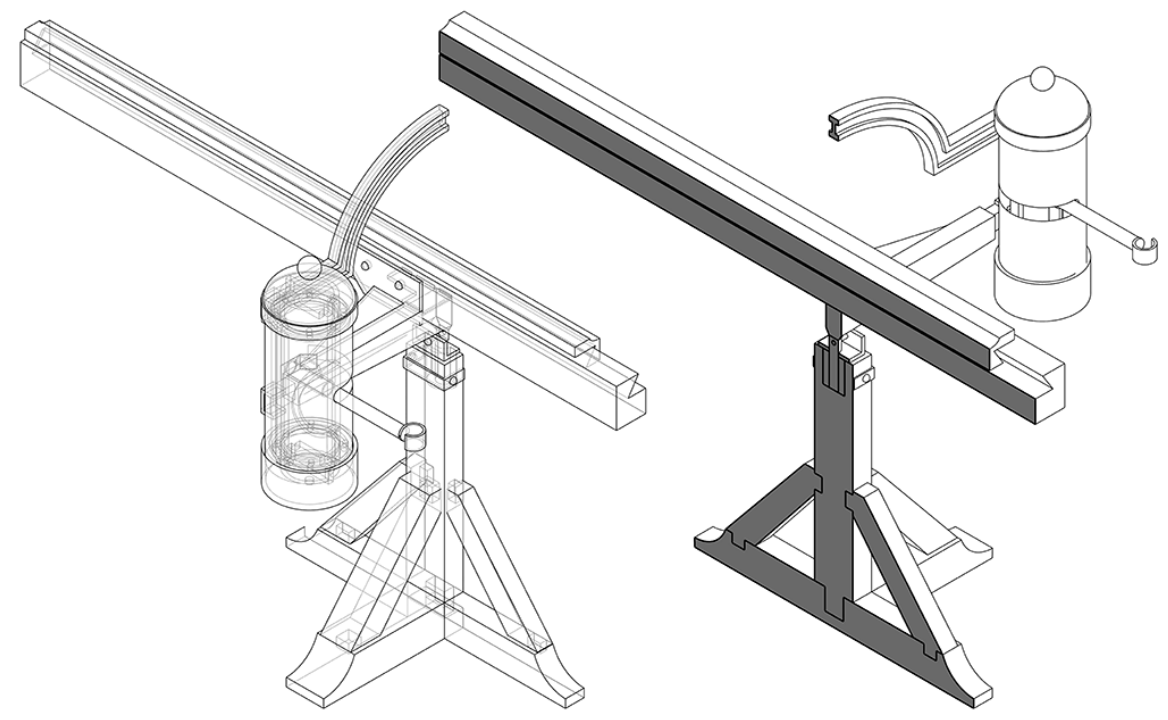

The reconstruction of the Trajan scorpion

The reconstruction of ancient war machines must show consistency with the rules of production, technological requirements, as well as with a series of dictates of a cultural nature. Every reconstruction, in fact, must be supported by a precise historical-archaeological reference and follow three essential criteria:

- be documented in written sources;

- have archaeological finds;

- refer to at least one iconographic mention.

The effectiveness of experimental archaeology activities is strongly linked to the control and correct interpretation of the archaeological finds at the basis of the reconstructions. Nevertheless, the executive design, the composition of the graphic tables, the re-elaboration of the executive details and the overall views are the basis of the entire operational process. From the acquisition of the sources to the executive definition of the components, the drawing is the main instrument of knowledge of the laws that govern the formal structure.

The project for the reconstruction of the Trajan Scorpio was initiated by the Archeotecnica working group in 2008. To support the executive design, the activities of the Laboratory of Advanced Representation Techniques of the Department of Engineering of the University of Campania "Luigi Vanvitelli" were aggregated. The research activity was pedagogically relayed on the didactic activity: the students had the opportunity to know the functioning of ballistic weaponry through the observation of the prototypes built materially in real scale, on the basis of drawings derived from the collected sources (figs. 2, 3, 4).

The assembly, with the concrete need to combine technical rules, functioning and historical coherence, has allowed the inductive confirmation of some of the questions posed and compared with other parallel studies. 
Fig. 9. Digital reconstruction ofTrajan Scorpio: renders (graphic elaboration by Umberto Palmieri).

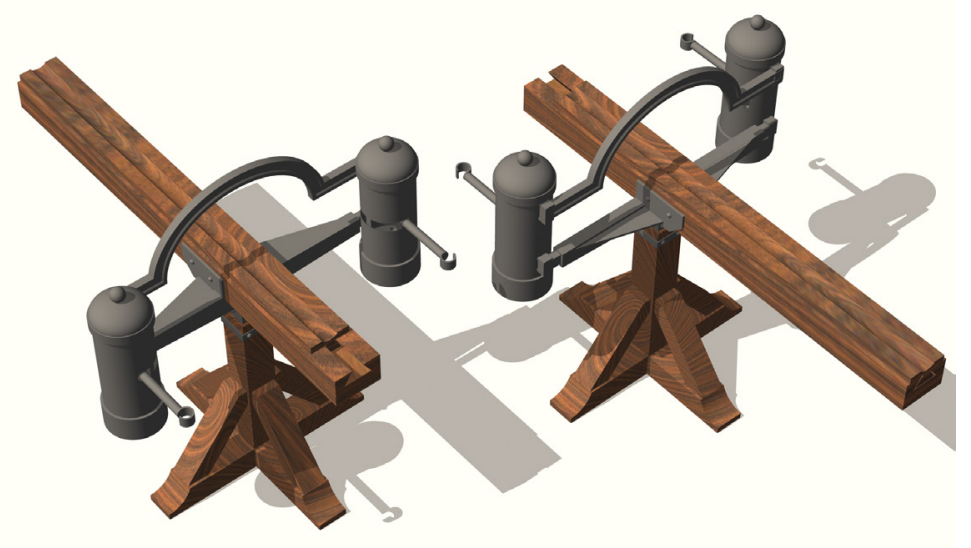

It is worth remembering that there are many typologies underlying the term 'relief'. In the broadest sense, this important process of analysis, which precedes any programmatic elaboration and idea of modification of the state of affairs, refers to the primitive action of removing from the context of interest what is peculiar in its forms and according to the particular objectives. The procedure, therefore, is implemented on the basis of the interest of the operator who can make use, in order to better carry it out, of adequate tools and precise methods (figs. 5-10).

The prodromal phase is therefore the acquisition of data which, when inherent to the scope of the architectural-topographical survey, insist on the measurement of nodal points. Compared to a reference system, the raised coordinates are considered objective, as they are repeatable data with the same initial conditions. It follows that surveying the measurements of a particular archaeological find constitutes the basis for its knowledge but, nevertheless, requires a shrewd geometrical interpretation to be translated into design opportunities. The restitution of the shape of a fragment, very often twisted and corroded, does not allow to answer with sufficient certainty the basic questions about what it was, or above all what it was not; what it was actually used for; and even more how it worked. In such circumstances it is necessary to proceed, as in the case of a complex identikit, relying on an indisputable mention in the literary sources, an evident proposition in the iconic sources (paintings, bas-reliefs, engravings, etc.) in which it is easily identifiable and the more or less similar archaeological finds certainly identified.
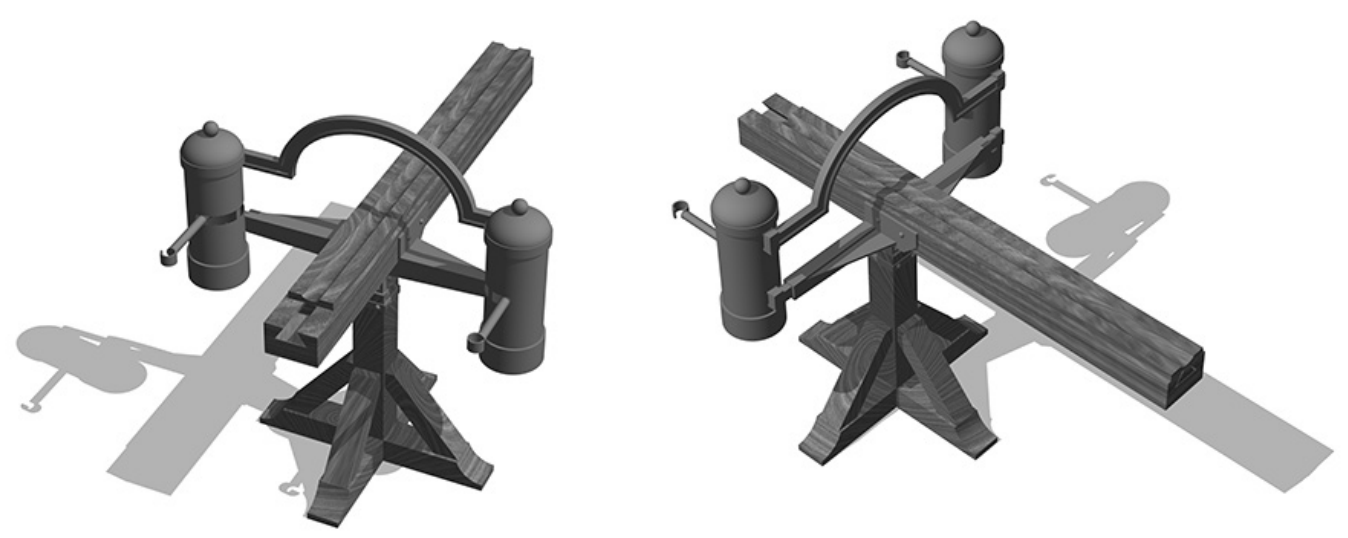
Fig. I I. 'Archotenica' by scorpion of Trajan; reconstruction serial number 13/19. Courtesy by Flavio Russo, the detal of 'campestria'.

Fig. 12. 'Archotenica' by Flavio Russo scorpion of Trajan; reconstruction serial number 13/19.

Courtesy by Flavio Russo, detail of the torsion motor.
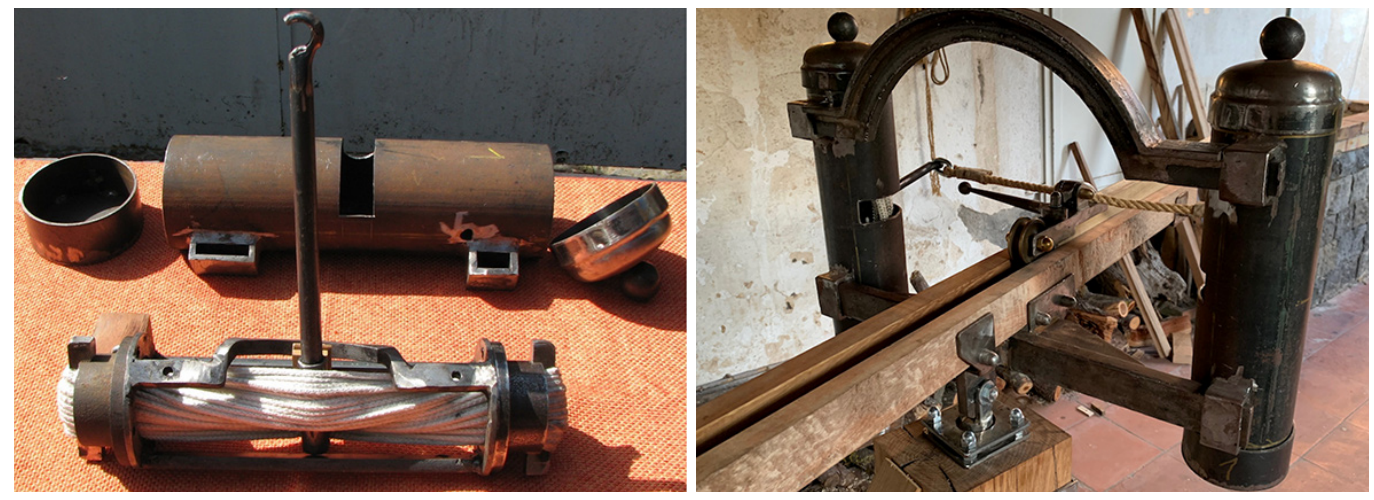

The correct reconstruction of the whole of belonging and origin, must therefore merge into the work of drawing in which the contemporary literary sources, iconic and material, are parameters of a model through which to articulate and switch the data in a process that converges in critical and design relief (figs. I I- |3).

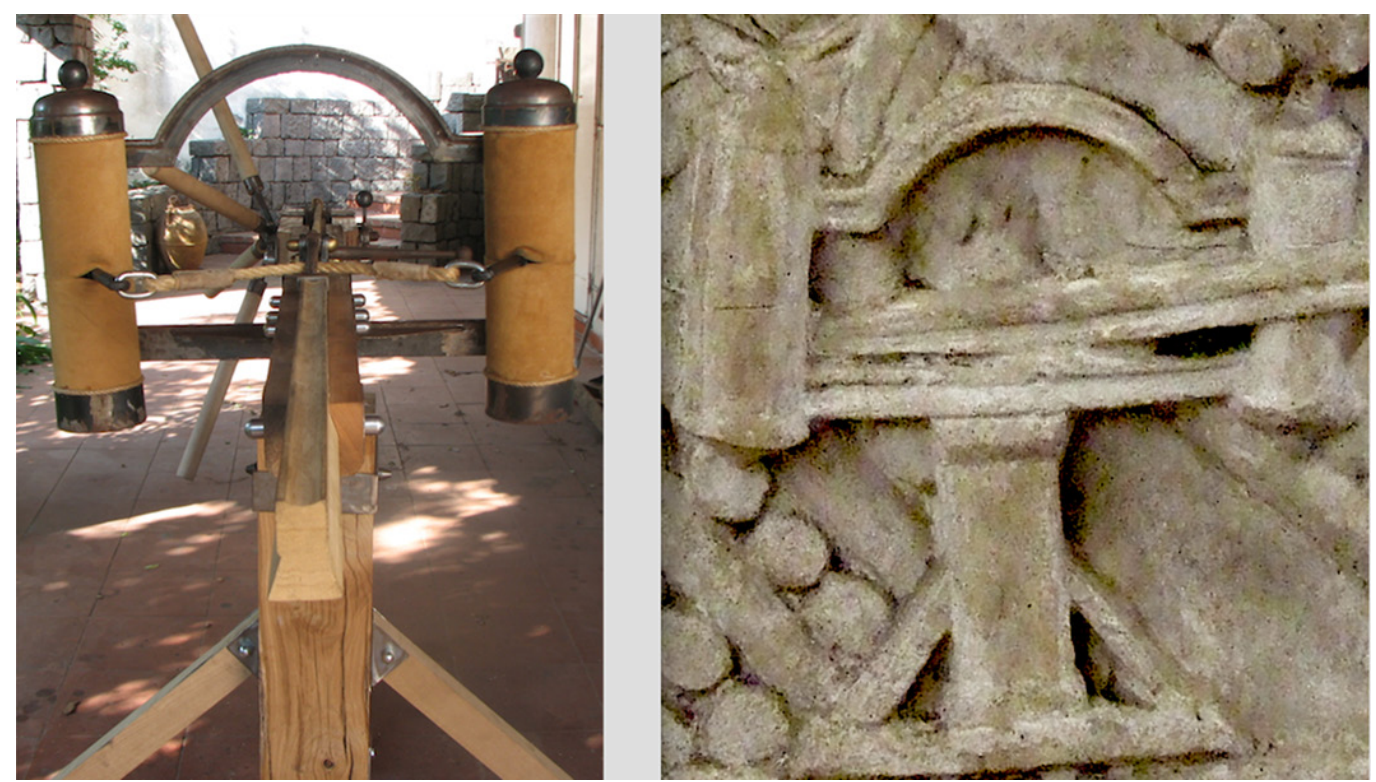

Notes

[I] The group - founded at the behest of Flavio Russo, a collaborator of the Army General Staff - includes, with different roles and skills, woodcutters, carpenters, blacksmiths, stonemasons, stonemasons, rope-makers who make, in addition to accessories, the raw parts of the machines assembled with the help of some motivated and patient collaborators. Adriana Rossi, full professor of Drawing (Lab. TAR), checks the correct digital modeling of the archaeological finds at the basis of the executive projects of the reconstructions.

[2] Velleio Patercolo, Storia romana (Historiae Romanae ad M. Vinicium libri duo), II, I I7- I20; Cassio Dione Cocceiano, Storia romana, LVI, I9-2I; Publio, Annio Floro, Epitome de T. Livio Bellorum omnium annorum DCC Libri duo, II, 36-37.

[3] ] Free translation: "You will tell new rivers flowing between lands, new bridges thrown over rivers, armies camped on cliffs, a king full of boldness hunted down, not from the throne, from life. And besides this, the magnificence of two triumphs, one of which was the first obtained over indomitable people, and the other the last". C. Plinii Caecilii Secundi, Epistolae, VII, 2.

[4] Marsden Eric William (197I). Greek and Roman Artillery. Technical Treatises. Oxford: Clarendon Press, pp. I05- I84; Marsden Eric William (1969). Greek and Roman Artillery, Historial Development. Oxford: Clarendon; Schramm Erwin (I9l8). Die Antiken Geschutze der Saalburg. Berlino 1918, rist. 1980. 


\section{References}

Referring to a more appropriate context the detailed description of the work carried out, we list the sources studied to perform the digital reconstructions of which the illustrated one is only a partial example:

Barbaro Daniele ( I567). I dieci libri dell'architettura di M. Vitruvio Tradotti \& commentati da Mons. Daniele Barbaro eletto Patriarca d'Aquileia, da lui riveduti \& ampliati. Venezia: Francesco de' Franceschi Senese. Roma: Bardi, 2006, facsimile.

Barbieri Nicola S. (1984). II primo trattato di tecnologia. La "Costruzione di armi da getto" di Filone di Bisenzio. Introduzione traduzione italiana e commento. Tesi di laurea (non pubblicata) in Lettere e Filosofia, relatore prof. G. A. Ferrari. Università degli Studi di Pavia.

Baatz Dietwulf (1974). Teile spatromischen Ballistenaus Gornea und Orşova (Rumanien). In Saalburg-Jahrbuch, 31 , pp. 50-72.

Baatz Dietwulf (1978). Recent Finds of Ancient Artillery. In Britannia, 9, pp. I- 17

Baatz Dietwulf, Feugere Michel (198I). Elements d'une catapulte romaine trouvee à Lyon. In Gallia, 39, pp. 201-209.

Campbell Duncan B. (2003). Greek and Roman Artillery 399 BC-AD 363. Oxford: Osprey Publishing.

Cesare Cesariano ( 152 I). Di Lucio Vitruvio Pollione De architectura libri dece traducti de latino in vulgare affigurati: commentati: \& con mirando ordine insigniti. Como: Gottardo da Ponte, facsimile. Traduzione di Cesare Cesariano.

Coccejano Cassio Dione (230). Storia romana. Libro LVI. Milano: BUR, I998, pp. I9-2I.

Codex Parisinus inter supplementa Greaca 607 (foll. 56r-58v). Paris: Bibliothèque Nationale, 1867 (Wescher) et 1906 (Schneider).

Coxeter Harold Scott Macdonald,Van de Craats Jan (1993). Philon lines in non-Euclidean planes. In Journal of Geometry, 48, I-2, pp. 26-55.

Diels Hermann, Schramm Erwin (1919). Philons Belopoiika (Abhandlungen der preussischen Akademie der Wissenschaften Philosoph. hist. KI. 16). Berlin: Reimer.

Diels Hermann, Schramm Erwin (1920). Exzerpte aus Philons Mechanik B. VII und VIII (vulgo funftes Buch) (Abhandlungen der preussischen Akademie der Wissenschaften, Philosoph. hist. KI. I 2). Berlin: Reimer

Drachmann Aage Gerhardt (1948). Ktesibios, Philon and Heron: A Study in Ancient Pneumatics. In Acta historica scientiarum naturalium et medicinalium, vol. 4.

Ferrari Gian Arturo (1982). Meccanica allargata. In Giannantoni Gabriele,Vegetti Mario (a cura di) Atti del convegno "La scienza ellenistica". Pavia I4-16 aprile 1982 pp. 227-296. Napoli: Bibliopolis.

Garlan Yvon (1972). La guerre dans l'antiquité. Bologna: II Mulino.

Garlan Yvon (1974). Recherches de poliorcetique grecque. Paris: Ecole francais d'Atenes.

Garlan Yvon (1973). Cités, armées et stratégie à l'époque hellénistique d'après l'oeuvre de Philonde Byzance. In Historia, 22 pp. 16-33.

Guzzomi Andrew Louis, Maraldi Mirko, Molari Pier Gabriele (2012). A historical review of the modulus concept and its relevance to mechanical engineering design today. In Mechanism and MachineTheory, 50, pp. I- I4.

Heronis di Alessandria (o il Vecchio) ( I 572). Liber de machinis bellicis, Venetiis, apud Franciscum Franciscium Senensem.

Landels John Gray ( 878$)$. Engineering in the ancient world. Berkeley: University of California Press.

Landrus Matthew (20 I0). Vinci's Giant Crossbow. Berlin: Springer.

MacDhnouill Gille, Nanzydon the Traveler (1918). A Modern Reconstruction of Vitruvius' Scorpion. Berlin.

Marsden Eric William (1969). Greek and Roman Artillery. Historical Development. Oxford: Clarendon Press.

Marsden Eric William ( 197I). Greek and Roman Artillery. Technical Treatises. Oxford: Clarendon Press, pp. I05- I84.

Molari Pier Gabriele et al. (20 I I). La ricostruzione della balista di Vitruvio. Esercitazioni dell'insegnamento Laboratorio di Storia dell'Ingegneria Meccanica tenuto da P.G. Molari nell' a.a. 20 I0-20I I. Facoltà di Ingegneria di Bologna.

Patercolo Velleio (30 d.C.). Storia romana (Historiae Romanae ad M. Vinicium libri duo), II, pp. I 17 - I 20

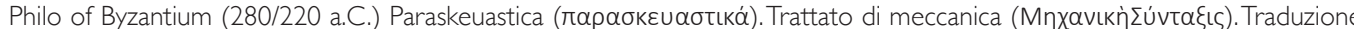
francese di Garlan Yvon (1974). Le livre "V" de la "syntaxe mecanique" de Pihilon de Byzance. In Recherches de poliorcétique grecque. Paris: Ecole Française d'Athenes De Boccard.

Prager Frank David (1974). Philo of Byzantium, Pneumatica: the first treatise on experimental physics, western version and eastern version. Historical Introduction and Technical Commentary by Wiesbaden Schone Richard (ed.) (1893). Berlin: Philonis Mechanicae Syntaxis, libri quartus et quintus.

Schmidt Wilhelm (ed.). ( 1899). Heronis Alexandrini opera I. Leipzig. pp. 458-89.

Publio Annio Floro (II sec. d.C.). Epitome de T. Livio Bellorum omnium annorum DCC Libri duo, II, 36-37. 
Rance Philip (20I3). Philo of Byzantium. In Roger S. Bagnall et al. (eds.). The Encyclopedia of Ancient History. Wiley-Blackwell; Chichester, E. Sussex/Malden, MA, pp. 5266-5268.

Rossi Adriana (20I5). Executive designs of a palinthytone stone-thrower in reconstructions of ancient Greco-Roman war machines. In <http://www.archeotecnica.com/chi-siamo>.

Rossi Lino (198I). Rotocalchi di pietra. Segni e disegni dei tempi sui monumenti trionfali dell'Impero Romano. Milano: Jaca Book.

Rossi Cesare, Russo Flavio, Russo Ferruccio (2009). Ancient engineers' inventions. Precursors of the present. Berlin: Springer.

Russo Flavio (2002). Tormenta, venti secoli di artiglieria meccanica. Roma: Stato Maggiore Esercito.

Russo Flavio (2004). La catapulta di Vitruvio. In Russo Flavio. L'artiglieria delle legioni romane. Roma: Libreria dello Stato, pp. I99280.

Schramm Erwin (1980). Die Antiken Geschutze der Saalburg. Berlino: Homburg.

Schone Richard (ed.) (1893). Philonis Mechanicae Syntaxis, libri quartus et quintus. Berlin.

Drachmann Aage Gerhardt (1948). Ktesibios, Philon and Heron. A study in ancient pneumatics. Copenhagen: E. Munksgaard.

Soedel Werner, Foley Vernard (1979). Ancient Catapults. In Scientific American, 3, 240, pp. I 50 - I 6 I.

Solís Santos Carlos (1996). Macchine, tecniche e meccanica. In Salvatore Settis (a cura di). Storia dei Greci e dei Romani, vol. 7 , pp. 723-24.

Taccola Mariano (I 38 | - 453 circa). De Ingeneis. vol. 2. Wiesbaden: Reichert Verlag. Facsimile.

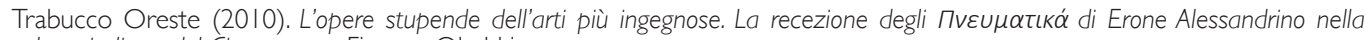
cultura italiana del Cinquecento. Firenze: Olschki.

Valturio Roberto ( 1472). De re militari. Biblioteca civica di Verona, inc.

Vitruvio Marco Pollione (1990). De Architectura - Libri X.Traduzione di Migotto Luciano. Pordenone: Ed. Studio Tesi.

Vitruvio Marco Pollione (2002). De Architectura - Libri X.Traduzione di Bossalino Franca. Roma: Edizioni Kappa.

Vitucci Giovanni (a cura di). (1974). La guerra giudaica. Milano: Mondadori.

Wescher Carl (20 I0). Poliorectique Des Grecs. Traites Theoriques, Recits Historiques. Whitefish: Kessinger Publishing.

Whitehead David (ed.) (1990). Aineias the Tactician. How to Survive Under Siege. Oxford: Clarendon Press.

Wilkins Alan (1995). Reconstructing cheirobbalista. In Journal of Roman Military Equipment Studies, 6, pp. 5-59.

Wilkins Alan (2003). Roman Artillery. Princes Risborough: Shire Publications.

Wilkins Alan, Morgan Len (2000). Scorpio and Cheiroballistra. In Journal of Roman Military Equipment Studies, I I, pp. 77- I 0 I.

\section{Authors}

Adriana Rossi, Università degli Studi della Campania “Luigi Vanvitelli”, adriana.rossi@unicampania.it

Umberto Palmieri, Università degli Studi della Campania “Luigi Vanvitelli”, umberto.palmieri@unicampania.it

To cite this chapter: Rossi Adriana, Palmieri Umberto (2020). Le immagini negate/The denied images. In Arena A., Arena M., Brandolino R.G. Colistra D., Ginex G., Mediati D., Nucifora S., Raffa P. (a cura di). Connettere. Un disegno per annodare e tessere. Atti del $42^{\circ}$ Convegno Internazionale dei Docenti delle Discipline della Rappresentazione/Connecting. Drawing for weaving relationships. Proceedings of the 42th International Conference of Representation Disciplines Teachers. Milano: FrancoAngeli, pp. 803-828 